NBER WORKING PAPER SERIES

\title{
EFFICIENCY AND WELFARE WITH COMPLEMENTARITIES AND ASYMMETRIC INFORMATION
}

\author{
George-Marios Angeletos \\ Alessandro Pavan \\ Working Paper 11826 \\ http://www.nber.org/papers/w11826 \\ NATIONAL BUREAU OF ECONOMIC RESEARCH \\ 1050 Massachusetts Avenue \\ Cambridge, MA 02138 \\ December 2005
}

\begin{abstract}
Earlier versions of this paper were entitled "Social Value of Coordination and Information." For stimulating discussions and useful comments, we thank Daron Acemoglu, Gadi Barlevy, Robert Barro, Olivier Blanchard, Marco Bassetto, Eddie Dekel, Christian Hellwig, Kiminori Matsuyama, Stephen Morris, Thomas Sargent, Hyun Song Shin, Ivàn Werning, and seminar participants at Harvard, MIT, Michigan, Northwestern, the Federal Reserve Bank of Chicago, the Bank of Italy, the 2005 workshop on beauty contests at the Isaac Newton Institute, and the 2005 workshop on coordination games at the Cowles Foundation. We are grateful to NSF for financial support (collaborative research grants SES-0519069 and SES-0518810) and to the Federal Reserve Bank of Minneapolis where part of this paper was completed. The views expressed herein are those of the author(s) and do not necessarily reflect the views of the National Bureau of Economic Research.
\end{abstract}

(C2005 by George-Marios Angeletos and Alessandro Pavan. All rights reserved. Short sections of text, not to exceed two paragraphs, may be quoted without explicit permission provided that full credit, including () notice, is given to the source. 
Efficiency and Welfare with Complementarities and Asymmetric Information

George-Marios Angeletos and Alessandro Pavan

NBER Working Paper No. 11826

December 2005

JEL No. C72, D62, D82

\begin{abstract}
This paper examines equilibrium and welfare in a tractable class of economies with externalities, strategic complementarity or substitutability, and incomplete information. In equilibrium, complementarity amplifies aggregate volatility by increasing the sensitivity of actions to public information; substitutability raises cross-sectional dispersion by increasing the sensitivity to private information. To address whether these effects are undesirable from a welfare perspective, we characterize the socially optimal degree of coordination and the efficient use of information. We show how efficient allocations depend on the primitives of the environment, how they compare to equilibrium, and how they can be understood in terms of a social trade-off between volatility and dispersion. We next examine the social value of information in equilibrium. When the equilibrium is efficient, welfare necessarily increases with the accuracy of information; and it increases [decreases] with the extent to which information is common if and only if agents' actions are strategic complements [substitutes]. When the equilibrium is inefficient, additional effects emerge as information affects the gap between equilibrium and efficient allocations. We conclude with a few applications, including production externalities, Keynesian frictions, inefficient fluctuations, and efficient market competition.
\end{abstract}

George-Marios Angeletos

Department of Economics

MIT

50 Memorial Drive, E51-251

Cambridge, MA 02142

and NBER

angelet@mit.edu

Alessandro Pavan

Northwestern University

2001 Sheridan

Arthur Andersen Hall 3239

Evanston, IL 60208

alepavan@northwestern.edu 


\section{Introduction}

In many economic environments, such as economies with production externalities, incomplete financial markets, or monopolistic competition, the action an agent wishes to take depends on his expectations not only about the underlying fundamentals but also about other agents' actions. Furthermore, different agents have different information about the fundamentals and hence different beliefs about what other agents are doing. Clearly, private incentives to coordinate and information asymmetries impact equilibrium behavior. But, are these incentives socially warranted, and is the decentralized use of information efficient? Also, does more precise information improve efficiency and welfare?

In this paper we examine equilibrium and welfare in a tractable class of concave economies that feature rich external and strategic effects - albeit a unique equilibrium - and asymmetric information.

There is a large number of ex-ante identical small agents each taking a continuous decision (e.g., investment). Individual payoffs may depend, not only on one's own action, but also on the mean, and possibly the dispersion, of activity in the population - this is the source of external and strategic effects in the model. Agents observe noisy private and public signals about the underlying economic fundamentals - this is the source of information asymmetry. Finally, payoffs are quadratic and information is Gaussian, which makes the analysis tractable.

In equilibrium, strategic complementarity raises the sensitivity of actions to public information; strategic substitutability raises the sensitivity to private information. Common noise in public information generates volatility; idiosyncratic noise in private information generates dispersion. It follows that complementarity contributes to higher volatility, substitutability to higher dispersion. ${ }^{1}$

These are interesting positive properties but alone have no normative content - there should be no presumption that the impact of strategic effects on the use of information and thereby on volatility and dispersion is undesirable from a welfare perspective. To address this issue, one needs to characterize the efficient use of information, which is our first main result.

We define efficient allocations as the ones that maximize ex-ante utility under the constraint that information can not be centralized. The efficient allocation for a given economy can be represented as the equilibrium of a fictitious economy where individual payoffs are manipulated to reflect social motives (that is, to internalize payoff interdependencies). The slope of best responses with respect to the mean activity in this fictitious economy measures the extent to which agents must align their choices for efficiency to obtain; it defines what we call the (socially) optimal degree of coordination. The analogue for the actual economy defines the equilibrium degree of coordination.

\footnotetext{
${ }^{1}$ The amplification effects of various sorts of complementarities are the subject of a vast literature. See Cooper (1990) for a review of complete-information applications and Morris and Shin $(2002,2003)$ for incomplete information.
} 
We first show how the efficient allocation depends on the primitives of the environment and how it compares to equilibrium allocation. As with equilibrium, complementarity contributes to a positive optimal degree of coordination, substitutability to a negative. But unlike equilibrium, the optimal degree of coordination also depends on other external payoff effects that are irrelevant for private incentives. In the absence of such non-strategic external effects, the optimal degree of coordination is higher than the equilibrium one when agents' actions are strategic complements (and lower when they are strategic substitutes).

This result highlights the danger in extrapolating positive properties to normative implications: in economies with complementarities, the high sensitivity to public information and the amplification of volatility featured in equilibrium can be socially desirable.

We next relate the optimal degree of coordination to the efficient use of information. Because of payoff concavity, both aggregate volatility and cross-sectional dispersion induce welfare losses. When comparing allocations that differ in their effective degree of coordination - and hence in their sensitivity to common and idiosyncratic noise - the planner effectively faces a trade-off between volatility and dispersion. The resolution of this trade-off is reflected in the optimal degree of coordination: the latter increases with social aversion to dispersion and decreases with social aversion to volatility.

Our second main result is a characterization of the social value of information. For this purpose, we find it useful to parameterize the information structure by the level and the composition of noise in the agents' forecasts of the underlying fundamentals. We identify the accuracy of available information with the precision of these forecasts, that is, the reciprocal of total noise, and its transparency (or commonality) with the correlation of forecast error across agents, that is, the extent to which noise is common. Since in the absence of external effects welfare depends only on the level and not on the composition of noise, this parametrization seems most appropriate from a theoretical point of view. ${ }^{2}$

When the equilibrium is efficient, welfare necessarily increases with the accuracy of information. Moreover, welfare increases [decreases] with the transparency of information if and only if agents' actions are strategic complements [substitutes]. Efficiency thus implies a clear relationship between the form of strategic interaction and the social value of information.

When the equilibrium is inefficient, information may also affect the gap between equilibrium and efficient allocations. Its welfare effects then depend, not only on the form of strategic interaction, but also on two key aspects of this gap: the discrepancy between optimal and equilibrium degrees of coordination, and the correlation between first-best and complete-information equilibrium activity.

\footnotetext{
${ }^{2}$ This parametrization is also appropriate for some applied questions. Think, for example, of a central banker contemplating whether to transmit information in a transparent or ambiguous way. This need not be simply a choice about the release of more or less information, but rather a choice about the extent to which individuals will adopt idiosyncratic or common interpretations of the same piece of information.
} 
We conclude the paper by illustrating how our results can help understand the inefficiencies of equilibrium and the social value of information in specific applications.

In a typical model of production spillovers where complementarities emerge in investment choices, coordination is inefficiently low. Moreover, welfare unambiguously increases with either the accuracy or the transparency of information - a case for timely provision of relevant information by the government or the media.

The same result appears to hold in standard Keynesian monetary economies. In contrast, coordination is inefficiently high and transparency can reduce welfare in economies resembling Keynes' beauty-contest parable for financial markets. Furthermore, in economies where equilibrium fluctuations are largely inefficient even under complete information, welfare may decrease with both accuracy and transparency - from a social perspective, ignorance could be a bless.

Finally, we consider an example of a competitive production economy where the equilibrium is efficient even under incomplete information. Since individual actions are strategic substitutes, welfare increases with accuracy but decreases with transparency-perhaps a case for "constructive ambiguity" in central bank communication.

Related literature. To the best of our knowledge, this paper is the first to conduct a complete welfare analysis for the class of economies considered here. The closest ascendants are Cooper and John (1988), who examine economies with complementarities but complete information, and Vives (1988), who shows efficiency of equilibria in a class of competitive economies that is a special case of the more general class considered here (see Section 6.4).

However, this paper is certainly not the first to examine the social value of information. Hirshleifer (1971) highlights how distributional effects can drive a wedge between the private and social value of information. More recently, and more closely related to this paper, Morris and Shin (2002) show that public information can reduce welfare in an economy that resembles a "beauty contest" and that features strategic complementarity. Angeletos and Pavan (2004) and Hellwig (2005), on the other hand, provide counterexamples where public information is socially valuable despite complementarity - a real economy with investment complementarities in the first paper, a monetary economy with pricing complementarities in the second. These works illustrate the non-triviality of the welfare effects of information within the context of specific applications, but do not explain the general principles underlying the question of interest. We fill the gap here by showing how the social value of information depends, not only on the form of strategic interaction, but also on other external effects that determine the discrepancy between equilibrium and efficient allocations.

The literature on rational expectations has emphasized how the aggregation of disperse private information in markets can improve allocative efficiency (e.g., Grossman, 1981). Laffont (1985) and Messner and Vives (2001), on the other hand, highlight how informational externalities can generate inefficiency in the private collection and use of information. Although the information 
structure here is exogenous, the paper provides an input into this line of research by studying how the welfare effects of private and public information depend on payoff externalities.

The paper also contributes to the debate about central-bank transparency. While earlier work focused on incentive problems (e.g., Canzoneri, 1985; Atkeson and Kehoe, 2001; Stokey, 2002), recent work emphasizes the coordinating role of public information. Morris and Shin $(2002,2005)$ and Heinemann and Cornand (2004) argue that central-bank disclosures can lead to welfare losses if markets behave like in Keynes' "beauty contest"; Svensson (2005) and Woodford (2005) question the practical relevance of this result; Hellwig (2005) and Roca (2005) show that public disclosures can improve welfare by reducing price dispersion. In Section 6.3 we highlight that the welfare effects of such disclosures ought to depend on whether the business cycle is efficient under complete information, while in Section 6.4 we show that an argument for constructive ambiguity could be made even in efficient competitive economies.

The rest of the paper is organized as follows. We introduce the model in Section 2. We examine equilibrium in Section 3, efficiency in Section 4, and the social value of information in Section 5. We turn to applications in Section 6. The Appendix includes proofs omitted in the main text.

\section{The model}

Actions and payoffs. Consider an economy with a measure-one continuum of agents, each choosing an action $k \in \mathbb{R}$. Let $\Psi$ denote the cumulative distribution function for $k$ in the crosssection of the population, $K \equiv \int k d \Psi(k)$ the mean action, and $\theta=\left(\theta_{1}, \ldots, \theta_{N}\right) \in \mathbb{R}^{N}$ a vector of exogenous payoff-relevant variables (the fundamentals), with $N \geq 1 .{ }^{3}$ Individual utility is given by

$$
u=U(k, K, \theta),
$$

where $U: \mathbb{R}^{N+2} \rightarrow \mathbb{R}$ is a strictly concave quadratic function. ${ }^{4,5}$ Finally, we let $W(K, \theta) \equiv$ $U(K, K, \theta)$ denote utility (also, aggregate welfare) when all agents choose the same action.

Externality emerges whenever $U_{K} \neq 0$, strategic complementarity whenever $U_{k K} \neq 0 .{ }^{6} \mathrm{We}$ restrict $-U_{k K} / U_{k k}$ within $(-1,+1)$. As we will see in the next section, $-U_{k K} / U_{k k}$ is the slope of best responses; restricting this slope within $(-1,+1)$ is necessary and sufficient for the existence of a unique stable equilibrium. We also impose concavity at both the individual and aggregate level in the sense that $U_{k k}<0$ and $W_{K K} \equiv U_{k k}+2 U_{k K}+U_{K K}<0$. If $U$ were not concave, best

\footnotetext{
${ }^{3}$ The analysis is simplest when $N=1$, but $N>1$ allow us capture the possibility that there are fundamentals that are relevant for equilibrium but not for efficient allocations, and vice versa.

${ }^{4}$ That is, $U(k, K, \theta)=v \mathbf{U} v^{\prime}$ where $\mathbf{U}$ is a $(n+3) \times(n+3)$ negative-definite matrix and $v=(1, k, K, \theta)$.

${ }^{5}$ Note that $U$ depends on $\Psi$ only through its first moment (mean activity); we extend the model to incorporate an external effect from the second moment (cross-sectional dispersion) at the end of Section 4.

${ }^{6}$ In what follows, we often refer to $U_{k K}$ as the complementarity even if $U_{k K}<0$. That is, we identify substitutability with negative complementarity.
} 
responses would not be well-defined; similarly, if $W$ were not concave, the first best would not be well-defined. ${ }^{7}$

Information. Before agents move, nature draws $\theta_{n}$, for $n \in\{1, \ldots, N\}$, from independent Normal distributions with mean $\mu_{n}$ and variance $\sigma_{\theta_{n}}^{2}$. The realization of $\theta=\left(\theta_{n}\right)$ is not observed by the agents. Instead, for each $n$, agents observe private signals $x_{n}^{i}=\theta_{n}+\xi_{n}^{i}$ and public signals $y_{n}=\theta_{n}+\varepsilon_{n}$, where $\xi_{n}^{i}$ and $\varepsilon_{n}$ are, respectively, idiosyncratic and common noises, independent of one another as well as of $\theta$, with variances, respectively, $\sigma_{x_{n}}^{2}$ and $\sigma_{y_{n}}^{2}$.

The common posterior for $\theta_{n}$ given public information alone is Normal with mean $z_{n} \equiv$ $\mathbb{E}\left[\theta_{n} \mid y\right]=\lambda_{n} y_{n}+\left(1-\lambda_{n}\right) \mu_{n}$ and variance $\sigma_{z_{n}}^{2}$, where $\lambda_{n} \equiv \sigma_{y_{n}}^{-2} / \sigma_{z_{n}}^{-2}$ and $\sigma_{z_{n}} \equiv\left(\sigma_{y_{n}}^{-2}+\sigma_{\theta_{n}}^{-2}\right)^{-1 / 2}$. In what follows, we often identify public information with $z$ rather than with $y .{ }^{8}$ Private posteriors, on the other hand, are Normal with mean $\mathbb{E}\left[\theta_{n} \mid x^{i}, y\right]=\left(1-\delta_{n}\right) x_{n}^{i}+\delta_{n} z_{n}$ and variance $\sigma_{n}^{2}$, where $\delta_{n} \equiv \sigma_{x_{n}}^{-2} / \sigma_{n}^{-2}$ and $\sigma_{n} \equiv\left(\sigma_{x_{n}}^{-2}+\sigma_{z_{n}}^{-2}\right)^{-1 / 2}$.

If we let $\omega_{n}^{i} \equiv \theta_{n}-\mathbb{E}\left[\theta_{n} \mid x^{i}, y\right]$ denote agent $i$ 's forecast error about $\theta_{n}$, then

$$
\sigma_{n}^{2}=\operatorname{Var}\left(\omega_{n}^{i}\right) \quad \text { and } \quad \delta_{n}=\operatorname{Corr}\left(\omega_{n}^{i}, \omega_{n}^{j}\right), i \neq j
$$

Hence, $\sigma_{n}$ measures the total noise in agents' forecasts about the fundamentals and $\delta_{n}$ the extent to which noise is common across agents. ${ }^{9}$ We accordingly identify the accuracy of information with $\sigma_{n}^{-2}$ and its transparency (or publicity) with $\delta_{n}$.

We prefer to parametrize the information structure by $\left(\delta_{n}, \sigma_{n}\right)$ rather than $\left(\sigma_{x_{n}}, \sigma_{y_{n}}\right)$ for two reasons. First, this is without any loss of generality since, given the prior, there is a one-to-one mapping between $\left(\sigma_{x_{n}}, \sigma_{y_{n}}\right)$ and $\left(\delta_{n}, \sigma_{n}\right)$ :

$$
\sigma_{n}^{-2}=\sigma_{x_{n}}^{-2}+\sigma_{y_{n}}^{-2}+\sigma_{\theta_{n}}^{-2}>0 \quad \text { and } \quad \delta_{n}=\frac{\sigma_{y_{n}}^{-2}+\sigma_{\theta_{n}}^{-2}}{\sigma_{x_{n}}^{-2}+\sigma_{y_{n}}^{-2}+\sigma_{\theta_{n}}^{-2}} \in(0,1) \text {. }
$$

Second, a change in $\sigma_{x_{n}}$ or $\sigma_{y_{n}}$ combines a change in the level of noise, $\sigma_{n}$, with a change in its composition, $\delta_{n}$. If there were no externalities and strategic interactions, welfare would depend only on $\sigma_{n}$, not $\delta_{n}$. With strategic interactions, instead, the extent to which information is public plays an important role since it affects the structure of higher order beliefs. From a theoretical point of view, it thus seems most interesting to separate these two effects. ${ }^{10}$

\footnotetext{
${ }^{7}$ For tractability, we have restricted $U$, and hence $W$, to be quadratic. For non-quadratic concave environments, our results represent approximations that are better the lower the noise in information. Convexities, on the other hand, may introduce effects about which our analysis is not appropriate. For example, aggregate convexities can generate a social value for lotteries.

${ }^{8}$ Throughout, we use the convenient vector notation $x=\left(x_{n}\right), y=\left(y_{n}\right)$, and similarly for all other variables. We also drop the superscript $i$ whenever it does not create confusion.

${ }^{9}$ Note that $\delta_{n}$ is also an increasing transformation of the relative precision of public information.

${ }^{10}$ In the context of specific applications, however, it is also interesting to translate the results in terms of comparative statics with respect to $\left(\sigma_{x}, \sigma_{z}\right)$. See Section 6 for some examples.
} 


\section{Equilibrium allocations}

Each agent chooses $k$ so as to maximize his expected utility, $\mathbb{E}[U(k, K, \theta) \mid x, y]$. The solution to this optimization problem gives the best response for the individual. The fixed point is the equilibrium.

Definition 1 An equilibrium allocation is any function $k: \mathbb{R}^{2 N} \rightarrow \mathbb{R}$ such that, for all $(x, y)$,

$$
k(x, y)=\arg \max _{k^{\prime}} \mathbb{E}\left[U\left(k^{\prime}, K, \theta\right) \mid x, y\right]
$$

where $K(\theta, \varepsilon)=\mathbb{E}[k(x, y) \mid \theta, \varepsilon]$, for all $(\theta, \varepsilon) \cdot{ }^{11}$

It is useful to consider first the complete-information benchmark. When $\theta$ is known, the (unique) equilibrium is $k=\kappa$, where $\kappa$ is the unique solution to $U_{k}(\kappa, \kappa, \theta)=0$. Since $U$ is quadratic, $\kappa$ is linear: $\kappa(\theta)=\kappa_{0}+\kappa_{1} \theta_{1}+\ldots+\kappa_{N} \theta_{N}$ for some constants $\kappa_{n} \in \mathbb{R}, n \in\{0,1, \ldots, N\} .{ }^{12}$ The incomplete-information equilibrium is then characterized as follows.

Proposition 1 Let $\kappa(\theta)=\kappa_{0}+\kappa_{1} \theta_{1}+\ldots+\kappa_{N} \theta_{N}$ denote the complete-information equilibrium allocation, $\bar{N} \equiv\left\{n \geq 1: \kappa_{n} \neq 0\right\} \neq \varnothing,{ }^{13}$ and

$$
\alpha \equiv \frac{U_{k K}}{\left|U_{k k}\right|} .
$$

(i) An allocation $k: \mathbb{R}^{2 n} \rightarrow \mathbb{R}$ is an equilibrium if and only

$$
k(x, y)=\mathbb{E}[(1-\alpha) \kappa+\alpha K \mid x, y] \text { for all }(x, y)
$$

where $K(\theta, \varepsilon)=\mathbb{E}[k(x, y) \mid \theta, \varepsilon]$.

(ii) The equilibrium exists, is unique, and is given by

$$
\begin{gathered}
k(x, y)=\kappa_{0}+\sum_{n \in \bar{N}} \kappa_{n}\left[\left(1-\gamma_{n}\right) x_{n}+\gamma_{n} z_{n}\right], \\
\gamma_{n}=\delta_{n}+\frac{\alpha \delta_{n}\left(1-\delta_{n}\right)}{1-\alpha\left(1-\delta_{n}\right)} \quad \text { for all } n \in \bar{N} .
\end{gathered}
$$

Proof. Part $(i)$. Take any strategy $k: \mathbb{R}^{2 N} \rightarrow \mathbb{R}$ and let $K(\theta, \varepsilon)=\mathbb{E}[k(x, y) \mid \theta, \varepsilon]$. A bestresponse is a strategy $k^{\prime}(x, y)$ that solves the first-order condition

$$
\mathbb{E}\left[U_{k}\left(k^{\prime}, K, \theta\right) \mid x, y\right]=0
$$

\footnotetext{
${ }^{11}$ A state of the world is given by the realizations of $\theta, \varepsilon$, and $\left\{\xi^{i}\right\}_{i \in[0,1]}$. However, since $\xi$ is i.i.d. across agents, $K$ and other aggregates are functions of $(\theta, \varepsilon)$ alone.

${ }^{12}$ Since $U$ is quadratic, $\kappa_{0}=-U_{k}(0,0,0) /\left(U_{k k}+U_{k K}\right)$ and $\kappa_{n}=-U_{k \theta_{n}} /\left(U_{k k}+U_{k K}\right), n \in\{1, \ldots, N\}$. It follows that $\kappa_{n} \neq 0$ if and only if $U_{k \theta} \neq 0$.

${ }^{13}$ The assumption $\bar{N} \neq \varnothing$ avoids the trivial case that the fundamentals are irrelevant for equilibrium.
} 
for all $(x, y)$. Using $U_{k}(\kappa, \kappa, \theta)=0$ for all $\theta$, and the fact that $U$ is quadratic, the best-response function must satisfy $\mathbb{E}\left[U_{k k}\left(k^{\prime}-\kappa\right)+U_{k K}(K-\kappa) \mid x, y\right]=0$, or equivalently,

$$
k^{\prime}(x, y)=\mathbb{E}[(1-\alpha) \kappa+\alpha K \mid x, y]
$$

for all $(x, y)$. In equilibrium, $k^{\prime}(x, y)=k(x, y)$, which gives $(4)$.

Part (ii). Since $\mathbb{E}[\kappa \mid x, y]$ is linear in $(x, z)$, it is natural to look for a fixed point that is linear in $x$ and $z$. Thus suppose the equilibrium is

$$
k(x, y)=a+b \cdot x+c \cdot z
$$

for some coefficients $a \in \mathbb{R}, b \in \mathbb{R}^{N}$ and $c \in \mathbb{R}^{N} \cdot{ }^{14}$ Then $K(\theta, \varepsilon)=a+b \cdot \theta+c \cdot z$ and therefore (4) reduces to

$$
k(x, y)=(1-\alpha) \kappa_{0}+\alpha a+((1-\alpha) \kappa+\alpha b) \cdot \mathbb{E}[\theta \mid x, y]+\alpha c \cdot z
$$

where $\kappa=\left(\kappa_{1}, \ldots, \kappa_{n}\right)$. Substituting $\mathbb{E}[\theta \mid x, y]=(\mathbf{I}-\boldsymbol{\Delta}) x+\boldsymbol{\Delta} z$, where $\mathbf{I}$ is the $N \times N$ identity matrix and $\boldsymbol{\Delta}$ is the $N \times N$ diagonal matrix with $n$-th element equal to $\delta_{n}$, we conclude that (7) is an equilibrium if and only if $a, b$ and $c$ solve

$$
a=(1-\alpha) \kappa_{0}+\alpha a, \quad b=(\mathbf{I}-\boldsymbol{\Delta})[(1-\alpha) \kappa+\alpha b], \quad \text { and } \quad c=\boldsymbol{\Delta}[(1-\alpha) \kappa+\alpha b]+\alpha c .
$$

Equivalently $a=\kappa_{0}, b_{n}=\kappa_{n}(1-\alpha)\left(1-\delta_{n}\right) /\left[1-\alpha\left(1-\delta_{n}\right)\right]$, and $c_{n}=\kappa_{n} \delta_{n} /\left[1-\alpha\left(1-\delta_{n}\right)\right]$, $n \in\{1, \ldots, N\}$. Note that $b_{n}+c_{n}=\kappa_{n}$ always; $b_{n}=c_{n}=0$ whenever $\kappa_{n}=0$; and $b_{n} \in\left(0, \kappa_{n}\right)$ and $c_{n} \in\left(0, \kappa_{n}\right)$ otherwise. Letting $\gamma_{n} \equiv c_{n} / \kappa_{n} \in(0,1)$ for any $n \in \bar{N}$ gives (5)-(6). Clearly, this is the unique linear equilibrium. Furthermore, since best responses are linear in $\mathbb{E}[\theta \mid x, y]$ and $\mathbb{E}[K \mid x, y]$, there do not exist equilibria other than this one. (This follows from the same argument as in Morris and Shin (2002); our payoffs are more general but the structure of beliefs and best responses is essentially the same.)

Condition (4) has a simple interpretation: an agent's best response is an affine combination of his expectation of some given "target" and his expectation of aggregate activity. The target is simply the complete-information equilibrium. The slope of best responses with respect to aggregate activity, $\alpha$, is what we identify with the equilibrium degree of coordination.

The sensitivity of the equilibrium allocation to private and public information depends on both the degree of coordination and the transparency of information. When $\alpha=0$, the weights on signals $x_{n}$ and $z_{n}$ are simply the Bayesian weights and hence $\gamma_{n}=\delta_{n}$. The term $\left[\alpha \delta_{n}\left(1-\delta_{n}\right)\right] /\left[1-\alpha\left(1-\delta_{n}\right)\right]$ thus measures the excess sensitivity of equilibrium allocations to public information as compared to the case where there are no complementarities. Note that this term is increasing in $\alpha$. Stronger complementarities thus lead to a higher relative sensitivity to public information. This is a direct

\footnotetext{
${ }^{14} \mathrm{~A}$ dot between two vectors denotes inner product.
} 
implication of the fact that, in equilibrium, public information is a relatively better predictor of aggregate behavior than private information. In other words, public information has also a coordinating role.

If information were complete $\left(\sigma_{n}=0\right.$ for all $n$, or at least for all $\left.n \in \bar{N}\right)$, all agents would choose $k=K=\kappa$. Incomplete information affects equilibrium behavior in two ways. First, common noise generates (non-fundamental) volatility, that is, variation in aggregate activity $K$ around the complete-information level $\kappa$. Second, idiosyncratic noise generates dispersion, that is, variation in the cross-section of the population. The first is measured by $\operatorname{Var}(K-\kappa)$, the second by $\operatorname{Var}(k-K)$. Their dependence on the degree of coordination and the information structure is characterized below. ${ }^{15}$

Proposition 2 (i) Volatility, $\operatorname{Var}(K-\kappa)$, necessarily increases with $\alpha$ and $\sigma_{n}$, and increases with $\delta_{n}$ if and only if $\alpha<0$ or $\delta_{n}<\frac{1-\alpha}{\alpha}$. Moreover, the impact of noise on volatility increases with $\alpha$ (i.e., $\left.\frac{\partial^{2} \operatorname{Var}(K-\kappa)}{\partial \sigma_{n} \partial \alpha}>0\right)$.

(ii) Dispersion, $\operatorname{Var}(k-K)$, necessarily decreases with $\alpha$ and $\delta_{n}$ and increases with $\sigma_{n}$. Moreover, the impact of noise on dispersion decreases with $\alpha$ (i.e., $\left.\frac{\partial^{2} \operatorname{Var}(k-K)}{\partial \sigma_{n} \partial \alpha}<0\right)$.

Higher complementarity thus mitigates the impact of noise on dispersion, and obtains a better alignment of individual choices, but amplifies aggregate volatility. Higher transparency also reduces dispersion possibly at the expense of higher volatility. Higher accuracy, on the other hand, reduces both volatility and dispersion. We will examine in more detail the welfare effects of information later. In the next section, we turn to the characterization of the efficient allocation and show how this relates to the optimal degree of coordination.

\section{Efficient allocations}

The property that complementarity generates high sensitivity to common noise, and thereby amplifies volatility, is interesting on its own. But this is only a positive property. To address the normative question of whether these effects are socially undesirable, one needs to understand what is the efficient use of information. We define efficient allocations as those that maximize ex-ante welfare (expected utility) among the ones that are measurable in the agents' decentralized information.

Definition 2 An efficient allocation is a function $k: \mathbb{R}^{2 N} \rightarrow \mathbb{R}$ that maximizes ex-ante utility

$$
\mathbb{E} u=\int_{(\theta, \varepsilon)} \int_{x} U(k(x, y), K(\theta, \varepsilon), \theta) d P(x \mid \theta, \varepsilon) d P(\theta, \varepsilon)
$$

\footnotetext{
${ }^{15}$ In the following, whenever we say "volatility" we mean volatility of aggregate activity around its completeinformation counterpart.
} 
subject to

$$
K(\theta, \varepsilon)=\int_{x} k(x, y) d P(x \mid \theta, \varepsilon), \text { for all }(\theta, \varepsilon) .
$$

where $P(\theta, \varepsilon)$ stands for the c.d.f. of the joint distribution of $(\theta, \varepsilon)$ and $P(x \mid \theta, \varepsilon)$ for the conditional distribution of $x$ given $\theta$ and $\varepsilon$.

We believe that this notion of efficiency is appropriate for the purposes of this paper. The allocation defined above is the solution to the "team problem" where agents choose a strategy cooperatively and commit to it. It thus answers exactly the question of interest for this paper, namely how allocations and welfare would change if agents were to internalize their payoff interdependences and appropriately adjust their use of available information. ${ }^{16}$ What is more, as we will see in Section 5, it is precisely this notion of efficiency that helps understand the social value of information in equilibrium.

We start by deriving a necessary and sufficient condition for efficient allocations.

Lemma 1 An allocation $k: \mathbb{R}^{2 N} \rightarrow \mathbb{R}$ is efficient if and only if, for almost all $(x, y)$,

$$
\mathbb{E}\left[U_{k}(k(x, y), K, \theta)+U_{K}(K, K, \theta) \mid x, y\right]=0,
$$

where $K(\theta, \varepsilon)=\mathbb{E}[k(x, y) \mid \theta, \varepsilon]$.

Proof. The Lagrangian of the problem in Definition 2 can be written as

$$
\begin{aligned}
\Lambda=\int_{(\theta, \varepsilon)} \int_{x} U(k(x, y), K(\theta, \varepsilon), \theta) d P( & x \mid \theta, \varepsilon) d P(\theta, \varepsilon)+ \\
& +\int_{(\theta, \varepsilon)} \lambda(\theta, \varepsilon)\left[K(\theta, \varepsilon)-\int_{x} k(x, y) d P(x \mid \theta, \varepsilon)\right] d P(\theta, \varepsilon) .
\end{aligned}
$$

The first order conditions for $K(\theta, \varepsilon)$ and $k(x, y)$ are therefore given by

$$
\begin{gathered}
\int_{x} U_{K}(k(x, y), K(\theta, \varepsilon), \theta) d P(x \mid \theta, \varepsilon)+\lambda(\theta, \varepsilon)=0 \quad \text { for almost all }(\theta, \varepsilon) \\
\int_{(\theta, \varepsilon)}\left[U_{k}(k(x, y), K(\theta, \varepsilon), \theta)-\lambda(\theta, \varepsilon)\right] d P(\theta, \varepsilon \mid x, y)=0 \quad \text { for almost all }(x, y)
\end{gathered}
$$

Noting that $U_{K}$ is linear in its arguments and that $K(\theta, \varepsilon)=\int_{x} k(x, y) d P(x \mid \theta, \varepsilon)$, condition (9) can be rewritten as $-\lambda(\theta, \varepsilon)=U_{K}(K(\theta, \varepsilon), K(\theta, \varepsilon), \theta)$. Replacing this into (10) gives (8). Since $U$ is strictly concave and the constraint is linear, (8) is both necessary and sufficient, which completes the proof.

\footnotetext{
${ }^{16}$ Our efficiency concept is the same as in Radner (1962) or Vives (1988) and shares with Hayek (1945) the idea that information is disperse and can not be communicated to a "center". Clearly, this is different from efficiency concepts that assume costless communication and focus on incentive constraints (e.g., Mirrlees, 1971; Holmstrom and Myerson, 1983).
} 
This result has a simple interpretation. The first-best allocation, which corresponds to the case where $\theta$ is commonly known and is henceforth denoted by $\kappa^{*}(\theta)$, maximizes $W(K, \theta) \equiv$ $U(K, K, \theta)$. It thus solves the the first-order condition $W_{K}(K, \theta)=0$, or equivalently $U_{k}(K, K, \theta)+$ $U_{K}(K, K, \theta)=0 .{ }^{17}$ The incomplete-information counterpart of this condition is (8).

We can then expand this condition to characterize the efficient allocation under incomplete information in a similar fashion as with equilibrium.

Proposition 3 Let $\kappa^{*}(\theta)=\kappa_{0}^{*}+\kappa_{1}^{*} \theta_{1}+\ldots+\kappa_{N}^{*} \theta_{N}$ denote the first-best allocation, $\bar{N}^{*} \equiv\{n \geq 1$ : $\left.\kappa_{n}^{*} \neq 0\right\} \neq \varnothing$, and

$$
\alpha^{*} \equiv \frac{2 U_{k K}+U_{K K}}{\left|U_{k k}\right|}=2 \alpha+\frac{U_{K K}}{\left|U_{k k}\right|} .
$$

(i) An allocation $k: \mathbb{R}^{2 N} \rightarrow \mathbb{R}$ is efficient if and only if

$$
k(x, y)=\mathbb{E}\left[\left(1-\alpha^{*}\right) \kappa^{*}+\alpha^{*} K \mid x, y\right] \quad \text { for almost all }(x, y),
$$

where $K(\theta, \varepsilon) \equiv \mathbb{E}[k(x, y) \mid \theta, \varepsilon]$.

(ii) The efficient allocation exists, is essentially unique, and is given by

$$
\begin{gathered}
k(x, y)=\kappa_{0}^{*}+\sum_{n \in \bar{N}^{*}} \kappa_{n}^{*}\left[\left(1-\gamma_{n}^{*}\right) x_{n}+\gamma_{n}^{*} z_{n}\right], \\
\gamma_{n}^{*}=\delta_{n}+\frac{\alpha^{*} \delta_{n}\left(1-\delta_{n}\right)}{1-\alpha^{*}\left(1-\delta_{n}\right)} \quad \text { for all } n \in \bar{N}^{*} .
\end{gathered}
$$

In equilibrium, each agent's action was an affine combination of his expectation of $\kappa$, the complete-information equilibrium action, and of his expectation of aggregate activity. The same is true here for the efficient allocation if we replace $\kappa$ with $\kappa^{*}$, the first-best action, and $\alpha$ with $\alpha^{*}$. In this sense, condition (12) is the analogue for efficiency of the best response for equilibrium. This idea is formalized by the following.

Proposition 4 Given an economy $\mathbf{e}=\left(U ; \sigma, \delta, \mu, \sigma_{\theta}\right) \in \mathcal{E}$, let $\mathcal{U}(\mathbf{e})$ be the set of functions $U^{\prime}$ such that, if agents perceived their payoffs to be $U^{\prime}$ rather than $U$, the equilibrium would coincide with the efficient allocation for $\mathbf{e}$.

(i) For every $\mathbf{e}, \mathcal{U}(\mathbf{e})$ is non-empty.

(ii) For every $\mathbf{e}, U^{\prime} \in \mathcal{U}(\mathbf{e})$ only if $\alpha^{\prime} \equiv-U_{k K}^{\prime} / U_{k k}^{\prime}$ equals $\alpha^{*}$.

Part (i) states that the efficient allocation can be represented as the equilibrium of a fictitious game where individual incentives are manipulated so as to coincide with social incentives. ${ }^{18}$ Part

\footnotetext{
${ }^{17}$ Since $U$ and hence $W$ is quadratic, $\kappa^{*}(\theta)=\kappa_{0}^{*}+\kappa_{1}^{*} \theta_{1}+\ldots+\kappa_{N}^{*} \theta_{N}$, where $\kappa_{0}^{*}=-W_{K}(0,0) / W_{K K}$ and $\kappa_{n}^{*}=$ $-W_{K \theta_{n}} / W_{K K}, n \in\{1, \ldots, N\}$. It follows that $\kappa_{n}^{*} \neq 0$ if and only if $W_{K \theta_{n}} \equiv U_{k \theta_{n}}+U_{K \theta_{n}} \neq 0$.

${ }^{18}$ In some cases, this may also suggest a way to implement the efficient allocation. For example, the government may be able to use taxes and subsidies to fashion individual best-responses.
} 
(ii), on the other hand, explains why we identify $\alpha^{*}$ with the optimal degree of coordination: $\alpha^{*}$ describes the level of complementarity that agents should perceive if the efficient allocation were to obtain as an equilibrium outcome, that is, if all externalities were to be internalized.

The counterpart of optimal coordination is the efficient use of information: the higher the optimal degree of coordination, the higher the sensitivity of efficient allocations to public information.

Corollary 1 The relative sensitivity of the efficient allocation to public information is higher than that of the equilibrium allocation if and only if the optimal degree of coordination is higher than the equilibrium one, which is turn is true if and only if the complementarity is high enough relative to second-order non-strategic effects:

$$
\gamma_{n}^{*} \geq \gamma_{n} \forall n \in \bar{N} \cap \bar{N}^{*} \quad \Longleftrightarrow \quad \alpha^{*} \geq \alpha \quad \Longleftrightarrow \quad U_{k K} \geq-U_{K K}
$$

Proposition 3 and Corollary 1 show how the efficient allocation depends on the primitives of the environment and how it compares to the equilibrium one. As with equilibrium, the optimal degree of coordination is increasing in the complementarity, $U_{k K}$. . But unlike equilibrium, the optimal degree of coordination depends also on $U_{K K}$, a second-order external effect that does not affect private incentives. In the absence of such an effect, the optimal degree of coordination is higher (in absolute value) than the equilibrium one $\left(\alpha^{*}=2 \alpha\right)$ reflecting the internalization of the externality generated by the complementarity.

To understand better the forces behind the determination of the optimal degree of coordination, an alternative representation is useful. Welfare (ex-ante utility) at the efficient allocation can be expressed as $\mathbb{E} u=\mathbb{E} W\left(\kappa^{*}, \theta\right)-\mathcal{L}^{*}$, where

$$
\mathcal{L}^{*}=\frac{\left|W_{K K}\right|}{2} \operatorname{Var}\left(K-\kappa^{*}\right)+\frac{\left|U_{k k}\right|}{2} \operatorname{Var}(k-K) .
$$

Note that $\mathbb{E} W\left(\kappa^{*}, \theta\right)$ is ex-ante utility in the first-best allocation, while $\mathcal{L}^{*}$ captures the welfare losses associated with incomplete information, namely those due to aggregate volatility and crosssectional dispersion. ${ }^{19}$

That volatility and dispersion generate welfare losses follows directly from concavity of preferences. Naturally, the weight on volatility is given by $W_{K K}$, the curvature of welfare with respect to aggregate activity, while the weight on dispersion is given by $U_{k k}$, the curvature of utility with respect to individual activity. Note that $W_{K K}=U_{k k}+2 U_{k K}+U_{K K}$. When there are no strategic and second-order external effects (in the sense that $U_{k K}=U_{K K}=0$ ), aggregate welfare inherits the curvature of individual utility $\left(W_{K K}=U_{k k}\right)$, so that volatility and dispersion contribute equally to welfare losses. Complementarity $\left(U_{k K}>0\right)$ helps offset the diminishing returns faced at the individual level, thus reducing concavity in the aggregate $\left(W_{K K}\right)$ and therefore the weight on volatility. The converse is true for substitutability $\left(U_{k K}<0\right)$ or external concavity $\left(U_{K K}<0\right)$.

\footnotetext{
${ }^{19}$ Condition (16) follows from a Taylor expansion around $k=K=\kappa^{*}(\theta)$; see the Appendix.
} 
Volatility is generated by common noise, dispersion by idiosyncratic noise. Increasing the relative sensitivity of allocations to public information - equivalently, raising the degree of coordinationdampens dispersion at the expense of higher volatility. The efficient use of information reflects the resolution of this trade-off.

Corollary 2 The optimal degree of coordination equals one minus the weight that welfare assigns to volatility relative to dispersion:

$$
\alpha^{*}=1-\frac{W_{K K}}{U_{k k}} .
$$

Extension. In some applications of interest, cross-sectional dispersion has a direct external effect on individual utility. For example, price dispersion has a negative effect on individual utility in New-Keynesian monetary models (see Hellwig, 2005). In the beauty contest of Morris and Shin (2002), on the other hand, dispersion has positive external effect (see Section 6.2).

We can easily accommodate such an effect - and we do so for the rest of the paper-provided that dispersion enters linearly in the utility function: $U\left(k, K, \theta, \sigma_{k}^{2}\right)$ with $U_{\sigma_{k}^{2}} \in \mathbb{R}$ being a constant and $\sigma_{k}^{2} \equiv \int(k-K)^{2} d \Psi(k)$. Then all our results go through once we replace the welfare weight on dispersion with $U_{k k}+2 U_{\sigma_{k}^{2}}{ }^{20}$ In particular, welfare is now given by $\mathbb{E} u=\mathbb{E} W\left(\kappa^{*}, \theta\right)-\mathcal{L}^{*}$, where

$$
\mathcal{L}^{*}=\frac{\left|W_{K K}\right|}{2} \operatorname{Var}\left(K-\kappa^{*}\right)+\frac{\left|U_{k k}+2 U_{\sigma_{k}^{2}}\right|}{2} \operatorname{Var}(k-K) .
$$

The optimal degree of coordination is

$$
\alpha^{*}=1-\frac{W_{K K}}{U_{k k}+2 U_{\sigma_{k}^{2}}}
$$

Finally condition (15) becomes

$$
\gamma_{n}^{*} \geq \gamma_{n} \forall n \in \bar{N} \cap \bar{N}^{*} \quad \Longleftrightarrow \quad \alpha^{*} \geq \alpha \quad \Longleftrightarrow \quad U_{k K} \geq-U_{K K}+2 U_{\sigma_{k}^{2}}
$$

Note that $\alpha^{*}$ is increasing in $U_{K K}$ (or $\eta$ ) and decreasing in $U_{\sigma_{k}^{2}}$ (or $\omega$ ). This is intuitive. A higher $U_{K K}$ decreases the social cost of volatility, while a higher $U_{\sigma_{k}^{2}}$ decreases the social cost of dispersion. Both these effects are external and non-strategic - they affect the social value of coordination without affecting private incentives. The former contributes to a higher optimal degree of coordination, the latter to a lower.

Efficient economies. We conclude this section with necessary and sufficient conditions for the equilibrium to be efficient under incomplete information.

\footnotetext{
${ }^{20}$ In analogy to $W_{K K}<0$, we impose $U_{k k}+2 U_{\sigma_{k}^{2}}<0$, which is necessary and sufficient for welfare to depend negatively on dispersion.
} 
Proposition 5 The equilibrium is efficient if and only if

$$
\kappa(\cdot)=\kappa^{*}(\cdot) \quad \text { and } \quad \alpha=\alpha^{*}
$$

or, equivalently, $U_{k K}+U_{K K}-2 U_{\sigma_{k}^{2}}=0, U_{K}(0,0,0)=U_{k}(0,0,0) U_{k K} / U_{k k}$, and $U_{K \theta_{n}}=U_{k \theta_{n}} U_{k K} / U_{k k}$ for all $n$.

The condition $\kappa(\cdot)=\kappa^{*}(\cdot)$ means that the equilibrium is efficient under complete information. But efficiency under complete information alone does not guarantee efficiency under incomplete information. What is also needed is efficiency in the use of information which obtains when in addition the equilibrium and the optimal degrees of coordination coincide. ${ }^{21}$

\section{Social value of information}

We now examine the impact of information on equilibrium welfare (allowing for $U_{\sigma_{k}^{2}} \neq 0$ ), starting with economies where the equilibrium is efficient. This provides a useful benchmark, not only because efficiency is always an excellent starting point, but also because in our class of economies efficiency implies a clear relation between the form of strategic interaction and the social value of information.

Proposition 6 Suppose the equilibrium is efficient. For any $n \in \bar{N}$, welfare necessarily decreases with $\sigma_{n}$, and increases [decreases] with $\delta_{n}$ if and only if agents' actions are strategic complements [substitutes].

As highlighted in the previous section, the impact of information on welfare at the efficient allocation is summarized in the impact of noise on volatility and dispersion (see condition (18)). An increase in $\sigma_{n}$ for given $\delta_{n}$ raises both volatility and dispersion and therefore necessarily reduces welfare. An increase in $\delta_{n}$ for given $\sigma_{n}$, on the other hand, is equivalent to a reduction in dispersion, possibly at the expenses of volatility. Such a substitution is welfare-improving if and only if the social cost of dispersion is higher than that of volatility, that is, if and only if $\alpha^{*}>0$.

Note that, when the equilibrium allocation is efficient, it maximizes ex-ante expected utility. That accuracy is beneficial can then be obtained also an implication of Blackwell's theorem. Indeed, the same observation implies that, when the equilibrium is efficient, welfare necessarily decreases with either $\sigma_{x_{n}}$ or $\sigma_{y_{n}}$, for any $n \in \bar{N}$.

Corollary 3 Suppose the equilibrium is efficient. Welfare necessarily increases with the precision of either private or public information.

\footnotetext{
${ }^{21}$ Note that $\alpha$ and $\alpha^{*}$ depend on $U$ but not on $(\sigma, \delta)$. This explains why efficiency can be checked on the basis of the payoff structure alone, as shown in Proposition 5 above.
} 
In economies where the equilibrium is inefficient, the welfare effects of information are more complicated for two reasons. First, the equilibrium degree of coordination need not coincide with the optimal one $\left(\alpha \neq \alpha^{*}\right)$, thus introducing inefficiency in the way the trade-off between volatility and dispersion is resolved. Second, the equilibrium level of activity may differ from the socially optimal one even under complete information $\left(\kappa \neq \kappa^{*}\right)$, thus introducing first-order welfare losses in addition to those associated with volatility and dispersion.

Consider first the role of $\alpha \neq \alpha^{*}$, maintaining for a moment $\kappa=\kappa^{*}$. The welfare losses associated with incomplete information continue to be the weighted sum of volatility and dispersion, as in (18). ${ }^{22}$ For given $\alpha$, a higher $\alpha^{*}$ means a lower relative weight on volatility and hence a lower cost associated with an increase in $\delta_{n} \cdot{ }^{23}$ It follows that, relatively to the efficiency benchmark (Proposition 6), inefficiently low coordination $\left(\alpha<\alpha^{*}\right)$ increases the social value of transparency, while inefficiently high coordination $\left(\alpha>\alpha^{*}\right)$ reduces it. On the other hand, the possibility that $\alpha \neq \alpha^{*}$ does not affect the value of accuracy: a lower $\sigma_{n}$ reduces both volatility and dispersion and therefore necessarily increases welfare.

Consider next the role of $\kappa \neq \kappa^{*}$, in which case the equilibrium is inefficient even under complete information. In equilibrium, welfare is given by $\mathbb{E} u=\mathbb{E} W(\kappa, \theta)-\mathcal{L}$, where

$$
\mathcal{L}=-\operatorname{Cov}\left(K-\kappa, W_{K}(\kappa, \theta)\right)+\frac{\left|W_{K K}\right|}{2} \cdot \operatorname{Var}(K-\kappa)+\frac{\left|U_{k k}+2 U_{\sigma_{k}^{2}}\right|}{2} \cdot \operatorname{Var}(k-K)
$$

are the welfare losses due to incomplete information. ${ }^{24}$ The last two terms in $\mathcal{L}$ are the familiar welfare losses associated with volatility and dispersion (second-order effects). The covariance term, on the other hand, captures a novel first-order effect. When the complete-information equilibrium is efficient $\left(\kappa=\kappa^{*}\right.$ and hence $\left.W_{K}(\kappa, \theta)=0\right)$, the covariance term is zero; this is merely an implication of the fact that small deviations around a maximum have zero first-order effects. But when the complete-information equilibrium is inefficient due to externalities $\left(W_{K}(\kappa, \theta) \neq 0\right)$, the covariance term contributes to a welfare loss or gain; this is because a positive [negative] correlation between $K-\kappa$, the "error" in aggregate activity due to incomplete information, and $W_{K}(\kappa, \theta)$, the social return to activity, mitigates [exacerbates] the first-order losses associated with externalities.

As shown in the Appendix (Proof of Proposition 7), this covariance term can be expressed as

$$
\operatorname{Cov}\left(K-\kappa, W_{K}(\kappa, \theta)\right)=\left|W_{K K}\right| \operatorname{Cov}\left(K-\kappa, \kappa^{*}-\kappa\right)=\left|W_{K K}\right| \sum_{n \in \bar{N}} \phi_{n} v_{n}
$$

\footnotetext{
${ }^{22}$ As obvious from the derivation of (16) in the Appendix, (16) and similarly (18) extend to $\alpha \neq \alpha^{*}$ as long as $\kappa=\kappa^{*}$. This can also be seen from (19) below noting that $W_{K}(\kappa, \theta)=0$ when $\kappa=\kappa^{*}$.

${ }^{23}$ Recall from Proposition 2 that volatility increases with $\delta_{n}$ if and only if $\alpha<0$ or $\delta<(1-\alpha) / \alpha$, which we assume here in order to simplify the discussion. In the alternative case, welfare necessarily increases with $\delta_{n}\left(\right.$ when $\left.\kappa=\kappa^{*}\right)$.

${ }^{24}$ Condition (19) follows from a Taylor expansion around $K=\kappa(\theta)$; see Appendix.
} 
where, for all $n \in \bar{N}$,

$$
\begin{aligned}
\phi_{n} & \equiv \frac{\kappa_{n}^{*}-\kappa_{n}}{\kappa_{n}}=\frac{\operatorname{Cov}\left(\kappa^{*}-\kappa, \kappa \mid \theta_{-n}\right)}{\operatorname{Var}\left(\kappa \mid \theta_{-n}\right)}, \\
v_{n} & \equiv-\frac{1}{1-\alpha+\alpha \delta_{n}} \kappa_{n}^{2} \sigma_{n}^{2}=\operatorname{Cov}\left(K-\kappa, \kappa \mid \theta_{-n}\right),
\end{aligned}
$$

with $\theta_{-n}$ standing for $\left(\theta_{j}\right)_{j \neq n}$. The coefficients $v_{n}$ capture the covariation between $K-\kappa$, the aggregate "error" due to incomplete information, and $\kappa$, the complete-information equilibrium, while the coefficients $\phi_{n}$ capture the covariation between the latter and $\kappa^{*}-\kappa$, the efficiency gap under complete information.

A lower $\sigma_{n}$ always implies a $v_{n}$ closer to zero, for less noise brings $K$ closer to $\kappa$ for any given $\theta$. But how this affects welfare depends on whether getting $K$ closer to $\kappa$ also means getting $K$ closer to $\kappa^{*}$, which in turn depends on the correlation between complete-information equilibrium and first best. Intuitively, less noise brings $K$ closer to $\kappa^{*}$ when $\phi_{n}>0$ but further away when $\phi_{n}<0$. As a result, the welfare contribution of a lower $\sigma_{n}$ through the covariance term in (19) is positive when $\phi_{n}>0$ but negative when $\phi_{n}<0$. Combining this with the effect of $\sigma_{n}$ on volatility and dispersion, we conclude that higher accuracy necessarily increases welfare when $\phi_{n}>0$ (i.e., when the correlation between equilibrium and first best is positive) but can reduce welfare when $\phi_{n}$ is sufficiently negative.

The impact of $\delta_{n}$ on $v_{n}$, on the other hand, depends on the sign of the complementarity: higher transparency increases the covariance between $K$ and $\kappa$ when $\alpha>0$ but decreases it when $\alpha<0$. How this in turn affects welfare depends again on the sign of $\phi_{n}$. Hence, as evident from (20), the sign of the effect of $\delta_{n}$ on first-order welfare losses depends on the sign of the product of $\alpha$ and $\phi_{n}$. Combining this with the effects of $\delta_{n}$ on volatility and dispersion, and noting that the covariance term dominates for $\phi_{n}$ sufficiently away from zero, we conclude that $\phi_{n}$ sufficiently high [low] suffices for the welfare effect of $\delta$ to have the same [opposite] sign as $\alpha$.

These insights are verified in the following complete characterization of the welfare effects of information.

Proposition 7 There exist functions $\underline{\phi}, \underline{\phi^{\prime}}, \bar{\phi}, \bar{\phi}^{\prime}:(-1,1) \times(-\infty, 1) \rightarrow \mathbb{R}$, with $\underline{\phi} \leq \bar{\phi}$ and $\underline{\phi}^{\prime} \leq$ $\bar{\phi}^{\prime}<0$, such that the following are true for any $n \in \bar{N}$ :

[Strategic Independece] When $\alpha=0$, welfare increases [decreases] with $\delta_{n}$ for all $\left(\sigma_{n}, \delta_{n}\right)$ if and only if $\alpha^{*}>0\left[\alpha^{*}<0\right]$.

[Strategic Complementarity] When $\alpha \in(0,1)$, welfare increases [decreases] with $\delta_{n}$ for all $\left(\sigma_{n}, \delta_{n}\right)$ if and only if $\phi_{n}>\bar{\phi}\left(\alpha, \alpha^{*}\right)\left[\phi_{n}<\underline{\phi}\left(\alpha, \alpha^{*}\right)\right]$.

[Strategic Substitutability] When $\alpha \in(-1,0)$, welfare increases [decreases] with $\delta_{n}$ for all $\left(\sigma_{n}, \delta_{n}\right)$ if and only if $\phi_{n}<\underline{\phi}\left(\alpha, \alpha^{*}\right)\left[\phi_{n}>\bar{\phi}\left(\alpha, \alpha^{*}\right)\right]$. 
[Accuracy] Welfare decreases [increases] with $\sigma_{n}$ for all $\left(\sigma_{n}, \delta_{n}\right)$ if and only if $\phi>\bar{\phi}^{\prime}\left(\alpha, \alpha^{*}\right)$ $\left[\phi<\underline{\phi}^{\prime}\left(\alpha, \alpha^{*}\right)\right]$

The functions $\underline{\phi}, \underline{\phi^{\prime}}, \bar{\phi}, \bar{\phi}^{\prime}$ are invariant with $\mathcal{E}$ and satisfy the following properties: $(i) \underline{\phi}=\underline{\phi}^{\prime}=\bar{\phi}=$ $\bar{\phi}^{\prime}=-\frac{1}{2}$ whenever $\alpha=\alpha^{*}$; (ii) for $\alpha \in(0,1), \underline{\phi}<0$ if and only if $\alpha>1 / 2$ or $\alpha^{*}>-\alpha^{2} /(1-2 \alpha)$, while $\bar{\phi}<0$ if and only if $\alpha^{*}>\alpha^{2}$; and (iii) for $\alpha \in(-1,0), \underline{\phi}<0$ if and only if $\alpha^{*}<\alpha^{2}$, while $\bar{\phi}<0$ if and only if $\alpha^{*}<-\alpha^{2} /(1-2 \alpha)$.

By Proposition 5, the equilibrium is efficient if and only if $\alpha=\alpha^{*}$ and $\kappa=\kappa^{*}$, in which case the welfare effects of information are given by 6 . If the only inefficiency is either that $\kappa_{0} \neq \kappa_{0}^{*}$ or that $\kappa_{n}^{*} \neq 0\left(=\kappa_{n}\right)$ for some $n \notin \bar{N}$, then this inefficiency does not affect the comparative statics of equilibrium welfare with respect to $\left(\delta_{n}, \sigma_{n}\right)$ for $n \in \bar{N}$; that is, Proposition 6 continues to hold for all $n \in \bar{N}$ as long as $\alpha=\alpha^{*}$ and $\phi_{n}=0$ for all $n \in \bar{N}$. Away from this benchmark, Proposition 5 implies that the social value of information can still be understood as a function of $\alpha, \alpha^{*}$, and $\left(\phi_{n}\right)_{n \in \bar{N}}$. We conclude that understanding the efficient use of information-which is what we did in the previous section - is also instrumental for understanding the social value of information.

The following sufficient conditions are then immediate for the case where $\kappa^{*}-\kappa$, the completeinformation efficiency gap, is either constant or positively correlated with $\kappa$.

Corollary 4 Suppose $\left(\phi_{n}\right)_{n \in \bar{N}} \geq 0$, in which case Cov $\left(\kappa^{*}-\kappa, \kappa\right) \geq 0$. Then, welfare always increases with the accuracy of information, whereas it increases with its transparency if $\alpha^{*} \geq \alpha>0$, and decreases with it if $\alpha^{*} \leq \alpha<0$.

The following case is also interesting, as it contrasts with the Blackwell-like result we encountered earlier for efficient economies.

Corollary 5 Suppose $\phi_{n}<-1 / 2$ and $\alpha=\alpha^{*}=0$. Welfare decreases with the precision of either private or public information about $\theta_{n}$.

\section{Applications}

In this section, we show how our results may help understand the relation between equilibrium and efficient allocations and the welfare effects of information in specific contexts of interest. For simplicity, in most cases we assume a single fundamental variable $(N=1)$ and drop the index $n$.

\subsection{Investment complementarities}

The canonical model of production externalities can be nested by interpreting $k$ as investment and defining individual payoffs as follows:

$$
U(k, K, \theta)=A(K, \theta) k-c(k),
$$


where $A(K, \theta)=(1-a) \theta+a K$ represents the private return to investment, with $a \in(0,1 / 2)$ and $\theta \in \mathbb{R}$, and $c(k)=k^{2} / 2$ the private cost of investment. ${ }^{25}$ Variants of this specification appear in Bryant (1983), Romer (1986), Matsuyama (1992), Acemoglu (1993), and Benhabib and Farmer (1994), as well as models of network externalities and spillovers in technology adoption. The important ingredient is that the private return to investment increases with the aggregate level of investment - the source of both complementarity and externality in this class of models.

The equilibrium level of investment under complete information is $\kappa=\theta$, whereas the first best is $\kappa^{*}=\frac{1-a}{1-2 a} \theta$, and hence $\phi=\frac{a}{1-2 a}>0$. That is, investment is inefficiently low for all $\theta>0$, and the more so the higher $\theta$. Furthermore, $U_{k k}=-1, U_{k K}=a>0$, and $U_{K K}=U_{\sigma_{k}^{2}}=0$. That is, there is a positive complementarity but no other second-order external effect. It follows that the equilibrium degree of coordination is positive and the optimal one is also positive and indeed higher that the equilibrium one: $\alpha=a>0$ and $\alpha^{*}=2 \alpha>\alpha>0$. Using this together with Corollary 4 , we have the following result.

Corollary 6 In the investment example described above, coordination is inefficiently low and welfare unambiguously increases with both the accuracy and the transparency of information.

In this example the agents' private desire to coordinate is, not only socially warranted, but actually not strong enough. It is then intuitive that higher transparency, or more precise public information, necessarily increases welfare by facilitating better coordination. ${ }^{26}$

Economies with frictions in financial markets - where complementarities emerge through collateral constraints, missing assets, or other types of market incompleteness - are often related to economies with investment complementarities like the one considered here. Although this is appropriate for many positive questions, it need not be so for normative purposes. As the examples we study in the next two sections highlight, the result here depends on the absence of certain secondorder external effects and on a sufficiently strong correlation between equilibrium and first-best activity. Whether these properties are shared by mainstream incomplete-market models is an open question.

\section{2 "Beauty contests" vs. other Keynesian frictions}

Keynes contended that financial markets often behave like "beauty contests" in the sense that traders try to forecast and outbid one another's forecasts, but this motive is (presumably) not

\footnotetext{
${ }^{25}$ This is the example we examined in Angeletos and Pavan (2004), although there we computed welfare conditional on $\theta$, thus omitting the effect of $\operatorname{Cov}(\kappa, K-\kappa)$ on welfare losses.

${ }^{26}$ Translating these results in terms of $\sigma_{x}$ and $\sigma_{y}$, it is easy to show that welfare unambiguously increases with a reduction in either $\sigma_{x}$ or $\sigma_{y}$. Hence, both public and private information are beneficial in this example. However, a higher $\alpha$, by increasing the value of transparency, increases the welfare gain of public information and decreases that of private information.
} 
warranted from a social perspective because it is due to some (unspecified) market imperfection. Capturing this idea with proper microfoundations is an open question, but one possible shortcut, following Morris and Shin (2002), is to define a "beauty-contest economy" as an economy in which $\alpha>0=\alpha^{*}$ and $\kappa(\cdot)=\kappa^{*}(\cdot)$. The first condition means that the private motive to coordinate is not warranted from a social perspective; the second means that the inefficiency of equilibrium vanishes as information becomes complete. By Proposition 7 we then have the following.

Corollary 7 In beauty-contest economies, welfare is increasing in accuracy but non-monotonic in transparency.

The specific payoff structure assumed by Morris and Shin (2002) is given by

$$
u_{i}=-(1-r) \cdot\left(k_{i}-\theta\right)^{2}-r \cdot\left(L_{i}-\bar{L}\right)
$$

where $\theta \in \mathbb{R}$ is the underlying fundamental, $L_{i}=L\left(k_{i}\right) \equiv \int\left(k^{\prime}-k_{i}\right)^{2} d \Psi\left(k^{\prime}\right)$ is the mean squaredistance of agent $i^{\prime}$ s action from other agents' actions, $\bar{L}=\int L(k) d \Psi(k)$ is the cross-sectional mean of $L_{i}$, and $r \in(0,1) .{ }^{27}$ This example is nested in our framework with ${ }^{28}$

$$
U\left(k, K, \theta, \sigma_{k}^{2}\right)=-(1-r) \cdot(k-\theta)^{2}-r \cdot(k-K)^{2}+r \cdot \sigma_{k}^{2} .
$$

It follows that $\kappa^{*}=\kappa=\theta, U_{k k}=-2, U_{k K}=2 r, U_{K K}=-2 r, U_{\sigma_{k}^{2}}=r$, and hence $\alpha=r>0=\alpha^{*}$.

Note how this example features two external effects that tilt the trade-off between volatility and dispersion in the opposite direction than the complementarity. In particular, $U_{K K}<0$ increases the social cost of volatility, while $U_{\sigma_{k}^{2}}>0$ decreases the social cost of dispersion. Both effects are non-strategic, in the sense that they do not affect private incentives, and both contribute to reducing the social value of coordination. In the specific example considered by Morris and Shin (2002), these effects perfectly offset the impact of the complementarity, so that the optimal level of coordination is zero - which explains why transparency, and thereby public information, can be welfare-reducing.

Keyensian frictions such as monopolistic competition or incomplete markets are in the heart of various macroeconomic complementarities (a.k.a. "multipliers" or "accelerators"). These frictions share with beauty contests the idea that complementarity originates in some market imperfection. However, the normative properties of beauty contests need not be shared by other Keynesian frictions.

\footnotetext{
${ }^{27}$ The first term in $u_{i}$ captures the value of taking an action close to a fundamental "target" $\theta$. The $L_{i}$ term introduces a private value for taking an action close to others' actions, whereas the $\bar{L}$ term ensures that there is no social value in doing so. Indeed, aggregating across agents gives $w=-(1-r) \int(k-\theta)^{2} d \Psi(k)$, so that, from a social perspective, it is as if utility were simply $u=-(k-\theta)^{2}$, in which case there is of course no social value to coordination.

${ }^{28}$ Note that $L_{i}=\int\left(\left(k^{\prime}-K\right)-\left(k_{i}-K\right)\right)^{2} d \Psi\left(k^{\prime}\right)=\left(k_{i}-K\right)^{2}+\sigma_{k}^{2}, \bar{L}=2 \cdot \sigma_{k}^{2}$, and $L_{i}-\bar{L}=\left(k_{i}-K\right)^{2}-\sigma_{k}^{2}$.
} 
Consider, for example, new-Keynesian monetary models where complementarity emerges in pricing decisions (e.g., Woodford, 2003; Hellwig, 2005; Lorenzoni, 2005; Roca, 2005). In this class of models, imperfect substitutability across goods results in a negative externality from crosssectional dispersion in prices $\left(U_{\sigma_{k}^{2}}<0\right)$, which in turn contributes to a higher optimal degree of coordination - the opposite of what happens in the beauty contest above. Hellwig (2005) provides an excellent analysis of this class of models. He shows that the optimal sensitivity to public information is higher than the equilibrium one. Moreover, the business cycle is efficient under complete information. Translating these properties in our framework gives $\alpha^{*}>\alpha>0$ and $\phi=0$, in which case, by Corollary 4, welfare increases with both accuracy and transparency. This helps understand why, unlike in Morris and Shin (2002), public information is welfare improving in Hellwig (2005) and Roca (2005).

\subsection{Inefficient fluctuations}

The focus in the previous section was on how the complementarity and second-order effects tilt the trade-off between volatility and dispersion. We now turn focus to first-order effects. In particular, we consider economies where the efficiency gap $\kappa^{*}-\kappa$ co-varies negatively with $\kappa$ - that is, economies where recessions are inefficiently deep.

To isolate the impact of first-order effects $(\phi \neq 0)$, we abstract from strategic and second-order external effects $\left(U_{k K}=U_{K K}=U_{\sigma_{k}^{2}}=0\right)$, so that $\alpha^{*}=\alpha=0$. From Proposition 7 then $\phi<-1 / 2$ is necessary and sufficient for welfare to decrease with accuracy and be independent of transparency.

Corollary 8 Suppose that $\alpha^{*}=\alpha=0$ and that equilibrium fluctuations are sufficiently inefficient in the sense that $\operatorname{Cov}\left(\kappa, \kappa^{*}\right)<\frac{1}{2} \operatorname{Var}(\kappa)$. Then welfare decreases with either private or public information.

As an example, consider an economy where $\theta=\left(\theta_{1}, \theta_{2}\right) \in \mathbb{R}^{2}$ and where agents engage in an investment activity without complementarity but for which private and social returns differ:

$$
U\left(k, K, \theta, \sigma_{k}^{2}\right)=\theta_{1} k-k^{2} / 2+\lambda\left(\theta_{2}-\theta_{1}\right) K
$$

for some $\lambda \notin(0,1)$. The private return to investment is $\theta_{1}$, while the social return is $\theta_{2} \neq \theta_{1}$. It follows that $\kappa=\theta_{1}$, while $\kappa^{*}=(1-\lambda) \theta_{1}+\lambda \theta_{2}$, and hence $\phi_{1}=-\lambda$. If $\lambda<1 / 2$, meaning that the discrepancy between private and social returns is small enough, then $\phi_{1}>-1 / 2$ and welfare increases with either private or public information about $\theta_{1}$. But if $\lambda<1 / 2$, meaning that the correlation between private and social returns is close to zero, then $\phi=-1<-1 / 2$ and welfare decreases with either private or public information about $\theta_{1}$. A special case of this is when $\lambda=1$ and $\sigma_{\theta_{2}}=0$, so that $\kappa^{*}$ is constant and the entire fluctuation in investment is inefficient. ${ }^{29}$

\footnotetext{
${ }^{29}$ If we maintain that the correlation between $\kappa$ and $\kappa^{*}$ is low enough but let $\alpha^{*}=\alpha>0$, then welfare continues to
} 
The recent debate on the merits of transparency in central bank communication has focused on the role of complementarities in new-Keynesian models (e.g., Morris and Shin, 2002; Svensson, 2005; Woodford, 2005; Hellwig, 2005; Roca, 2005). The results of this and the previous section suggest that this debate might be somewhat misfocused - a critical role is played by the inefficiency of equilibrium fluctuations.

For example, we conjecture that the result in Hellwig (2005) and Roca (2005) that public information has a positive effect on welfare relies on the property that the business cycle is efficient in these models. In standard new-Keynesian models (e.g., Woodford, 2003) the monopolistic markup introduces an efficiency gap. As long fluctuations are driven by productivity, taste, or monetary shocks - which is the case in Hellwig (2005) and Roca (2005) - this gap remains constant over the business cycle. But if the business cycle is driven by shocks in mark-ups or the "labor wedge," it seems possible that providing markets with information that helps predict these shocks can reduce welfare. This is an interesting question that we leave open for future research.

\subsection{Efficient competitive economies}

The examples considered so far feature either positive complementarity or some form of inefficiency. We now turn to competitive economies where agents' choices are strategic substitutes and where the equilibrium is efficient under both complete and incomplete information. ${ }^{30}$

There is a continuum of households, each consisting of a consumer and a producer, and two commodities. Let $q_{1 i}$ and $q_{2 i}$ denote the respective quantities purchased by consumer $i$ (the consumer living in household $i$ ). His preferences are given by

$$
u_{i}=v\left(q_{1 i}, \theta\right)+q_{2 i}
$$

where $v(q, \theta)=\theta q-b q^{2} / 2, \theta \in \mathbb{R}$, and $b>0$, while his budget is

$$
p q_{1 i}+q_{2 i}=e+\pi_{i}
$$

where $p$ is the price of good 1 relative to good $2, e$ is an exogenous endowment of good 2 , and $\pi_{i}$ are the profits of producer $i$ (the producer living in household $i$ ), which are also denominated in terms of good 2. Profits in turn are given by

$$
\pi_{i}=p k_{i}-c\left(k_{i}\right)
$$

decrease with accuracy but now it also decreases with transparency - which strengthens particularly the case against public information.

${ }^{30}$ We constructed this class of quadratic competitive economies independently but then found out that Vives (1988) had proved efficiency of equilibria for exactly this class long before us. Hence, with regard to this particular class, only the welfare effects of information are novel here. 
where $k_{i}$ denotes the quantity of good 1 produced by household $i$ and $c(k)$ the cost in terms of good 2 , with $c(k)=k^{2} / 2 .^{31}$

The random variable $\theta$ represents a shock in the relative demand for the two goods. Exchange and consumption take place once $\theta$ has become common knowledge. On the contrary, production takes place at an earlier stage, when information is still incomplete.

Consumer $i$ chooses $\left(q_{1 i}, q_{2 i}\right)$ so as to maximize (22) subject to (23), which gives $p=\theta-b q_{1 i}$. Clearly, all households consume the same quantity of good 1, which together with market clearing gives $q_{1 i}=K$ for all $i$ and $p=\theta-b K$, where $K=\int k d \Psi(k)$. It follows that $i$ 's utility can be restated as $u_{i}=v(K, \theta)-p K+e+\pi_{i}=b K^{2} / 2+e+\pi_{i}$, where $\pi_{i}=p k_{i}-c\left(k_{i}\right)=(\theta-b K) k_{i}-k_{i}^{2} / 2$. This example is thus nested in our model with

$$
U\left(k, K, \theta, \sigma_{k}^{2}\right)=(\theta-b K) k_{i}-k_{i}^{2} / 2+b K^{2} / 2+e,
$$

in which case $\kappa^{*}=\kappa=\theta /(1+b), U_{k k}=-1, U_{k K}=-b, U_{K K}=b, U_{\sigma_{k}^{2}}=0$, and therefore $\phi=0$ and $\alpha^{*}=\alpha=-b<0$.

That the complete-information equilibrium is efficient $\left(\kappa=\kappa^{*}\right)$ should not be a surprise. Under complete information, the economy is merely an example of a complete-markets competitive economy in which the first welfare theorem applies. What is interesting is that the equilibrium remains (constrained) efficient under incomplete information, despite the absence of ex-ante complete markets. This is because the strategic substitutability perceived by the agents coincides with the one that the planner would have liked them to perceive $\left(\alpha^{*}=\alpha\right){ }^{32}$ The following is then a direct implication of Proposition 6.

Corollary 9 In the competitive economy described above, the equilibrium is efficient and welfare unambiguously decreases with both $\delta$ and $\sigma$.

This result may be relevant for the debate on transparency vs. constructive ambiguity in monetary policy. If we interpret "transparent" central bank disclosures as information that admits a single common interpretation and "ambiguous" disclosures as information that admits multiple idiosyncratic interpretations, then the result above makes a case for constructive ambiguity. This may be reminiscent of Morris and Shin (2002), but is different. Whereas the result there was driven by inefficiently high coordination $\left(\alpha^{*}=0<\alpha\right)$, here it is due to efficient substitutability $\left(\alpha^{*}=\alpha<0\right)$. It is perhaps more surprising that a case for constructive ambiguity can be made even for efficient competitive economies.

\footnotetext{
${ }^{31}$ Implicit behind this cost function is a quadratic production frontier. The resource constraints are therefore given by $\int_{i} q_{1 i}=\int_{i} k_{i}$ and $\int_{i} q_{2 i}=e-\frac{1}{2} \int_{i} k_{i}^{2}$ for good 1 and 2 , respectively.

${ }^{32}$ The equilibrium would be inefficient if we had defined welfare as producer surplus alone, which may be relevant for open economies that are net exporters of good 2. In this case, $u_{i}=\pi_{i}$ and therefore $\alpha^{*}=-2 b<\alpha=-b<0$; that is, the cooperative solution between the producers would involve stronger substitutability (and hence less sensitivity to public information) than equilibrium.
} 
Finally, this last result opens up the possibility that the informative role of prices may be welfare damaging even in competitive economies where the use of information is efficient. For example, suppose that an exogenous increase in the informativeness of prices - caused for example by a reduction in the impact of noisy traders in financial markets-leads agents to reduce their costly collection of private information. Due to strategic substitutability, such a substitution of private information for public information could reduce welfare even if agents' overall uncertainty reduces. ${ }^{33}$

Clearly, the model in this paper does not allow for information aggregation through prices, but extending the results in this direction seems a promising line for future research.

\section{Appendix}

Proof of Proposition 2. From condition (5),

$$
\begin{aligned}
k(x, y) & =\kappa_{0}+\sum_{n \in \bar{N}} \kappa_{n}\left[\left(1-\gamma_{n}\right) x_{n}+\gamma_{n} z_{n}\right] \\
K(\theta, \varepsilon) & =\kappa_{0}+\sum_{n \in \bar{N}} \kappa_{n}\left[\left(1-\gamma_{n}\right) \theta_{n}+\gamma_{n} z_{n}\right]
\end{aligned}
$$

Hence $k-K=\sum_{n \in \bar{N}} \kappa_{n}\left[\left(1-\gamma_{n}\right)\left(x_{n}-\theta_{n}\right)\right]$ and $K-\kappa=\sum_{n \in \bar{N}} \kappa_{n} \gamma_{n}\left(z_{n}-\theta_{n}\right)$. Using $\operatorname{Var}\left(x_{n}-\theta_{n}\right)=\sigma_{x_{n}}^{2}, \operatorname{Var}\left(z_{n}-\theta_{n}\right)=\sigma_{z_{n}}^{2}=\left(\sigma_{y_{n}}^{-2}+\sigma_{\theta_{n}}^{-2}\right)^{-1}$ and $\delta_{n}=\sigma_{z_{n}}^{-2} / \sigma_{n}^{-2}$, together with (6), we have

$$
\begin{aligned}
\operatorname{Var}(k-K) & =\sum_{n \in \bar{N}} \kappa_{n}^{2}\left[\left(1-\gamma_{n}\right)^{2} \sigma_{x_{n}}^{2}\right]=\sum_{n \in \bar{N}} \kappa_{n}^{2} \frac{(1-\alpha)^{2}\left(1-\delta_{n}\right)}{\left(1-\alpha+\alpha \delta_{n}\right)^{2}} \sigma_{n}^{2} \\
\operatorname{Var}(K-\kappa) & =\sum_{n \in \bar{N}} \kappa_{n}^{2} \gamma_{n}^{2} \sigma_{z_{n}}^{2}=\sum_{n \in \bar{N}} \kappa_{n}^{2}\left[\frac{\delta_{n}}{\left(1-\alpha+\alpha \delta_{n}\right)^{2}} \sigma_{n}^{2}\right]
\end{aligned}
$$

which gives the result.

Proof of Proposition 3. Part (i). Since $U$ is quadratic, (8) can be rewritten as

$$
\begin{aligned}
\mathbb{E}\left[U_{k}\left(\kappa^{*}, \kappa^{*}, \theta\right)+U_{k k} \cdot\left(k(x, y)-\kappa^{*}\right)\right. & +U_{k K} \cdot\left(K-\kappa^{*}\right)+ \\
& \left.+U_{K}\left(\kappa^{*}, \kappa^{*}, \theta\right)+\left(U_{k K}+U_{K K}\right) \cdot\left(K-\kappa^{*}\right) \mid x, y\right]=0 .
\end{aligned}
$$

\footnotetext{
${ }^{33}$ Of course, for this to be true it must be that there is some inefficiency in the collection of information, or otherwise Blackwell's theorem would again imply that any exogenous information is beneficial.
} 
Using $W_{K}\left(\kappa^{*}, \theta\right)=U_{k}\left(\kappa^{*}, \kappa^{*}, \theta\right)+U_{K}\left(\kappa^{*}, \kappa^{*}, \theta\right)=0$, the above reduces to

$$
\mathbb{E}\left[U_{k k}\left(k(x, y)-\kappa^{*}\right)+\left(2 U_{k K}+U_{K K}\right)\left(K-\kappa^{*}\right) \mid x, y\right]=0
$$

which together with $W_{K K}=U_{k k}+2 U_{k K}+U_{K K}$ gives (12).

Part (ii) follows from the same steps as in the proof of Proposition (1) replacing $\alpha$ with $\alpha^{*}$ and $\kappa(\cdot)$ with $\kappa^{*}(\cdot)$.

Proof of Proposition 4. Consider first part (ii). When agents perceive payoffs to be $U^{\prime}$, the equilibrium is the unique function $k: \mathbb{R}^{2 N} \rightarrow \mathbb{R}$ that solves

$$
k(x, y)=\mathbb{E}\left[\left(1-\alpha^{\prime}\right) \kappa^{\prime}+\alpha^{\prime} K \mid x, y\right]
$$

for all $(x, y) \in \mathbb{R}^{2 N}$, where $K(\theta, \varepsilon)=\mathbb{E}[k(x, y) \mid \theta, \varepsilon], \alpha^{\prime} \equiv-U_{k K}^{\prime} / U_{k k}^{\prime}$ and $\kappa^{\prime}(\theta)=\kappa_{0}^{\prime}+\kappa_{1}^{\prime} \theta_{1}+$ $\ldots+\kappa_{N}^{\prime} \theta_{N}$ is the unique solution to $U_{k}^{\prime}\left(\kappa^{\prime}, \kappa^{\prime}, \theta\right)=0$. From the same arguments as in the proof of Proposition 1 , the unique solution to $(25)$ is given by

$$
k(x, y)=\kappa_{0}^{\prime}+\sum_{n \in \bar{N}^{\prime}} \kappa_{n}^{\prime}\left[\left(1-\gamma_{n}^{\prime}\right) x_{n}+\gamma_{n}^{\prime} z_{n}\right]
$$

where

$$
\gamma_{n}^{\prime}=\delta_{n}+\frac{\alpha^{\prime} \delta_{n}\left(1-\delta_{n}\right)}{1-\alpha^{\prime}\left(1-\delta_{n}\right)} \quad \forall n \in \bar{N}^{\prime} \equiv\left\{n \geq 1: \kappa_{n}^{\prime} \neq 0\right\} \neq \varnothing
$$

For this to coincide with the efficient allocation for all $(x, y) \in \mathbb{R}^{2 N}$, it is necessary and sufficient that $\kappa^{\prime}(\cdot)=\kappa^{*}(\cdot)$ and that $\alpha^{\prime}=\alpha^{*}$, which proves part (ii).

For part (i) it suffices to let $U^{\prime}(k, K, \theta)=U(k, K, \theta)+U_{K}(K, K, \theta) k$, in which case it is immediate that $\kappa^{\prime}(\cdot)=\kappa^{*}(\cdot)$, and $\alpha^{\prime}=\alpha^{*}$.

Proof of Condition (16). Since $U$ is quadratic, a second-order Taylor expansion around $k=K$ is exact:

$$
U(k, K, \theta)=U(K, K, \theta)+U_{k}(K, K, \theta) \cdot(k-K)+\frac{U_{k k}}{2} \cdot(k-K)^{2} .
$$

It follows that ex-ante utility is given by

$$
\mathbb{E} u=\mathbb{E}[W(K, \theta)]+\frac{U_{k k}}{2} \mathbb{E}\left[(k-K)^{2}\right],
$$

where $k=k(x, y)$ and $K=K(\theta, \varepsilon)$ are shortcuts for the efficient allocation and $W(K, \theta) \equiv$ $U(K, K, \theta)$. A quadratic expansion of $W(K, \theta)$ around $\kappa^{*}$, which is exact since $U$ and thus $W$ are quadratic, gives

$$
W(K, \theta)=W\left(\kappa^{*}, \theta\right)+W_{K}\left(\kappa^{*}, \theta\right) \cdot\left(K-\kappa^{*}\right)+\frac{W_{K K}}{2} \cdot\left(K-\kappa^{*}\right)^{2} .
$$


By definition of $\kappa^{*}, W_{K}\left(\kappa^{*}, \theta\right)=0$. It follows that

$$
\mathbb{E} u=\mathbb{E} W\left(\kappa^{*}, \theta\right)+\frac{W_{K K}}{2} \cdot \mathbb{E}\left[\left(K-\kappa^{*}\right)^{2}\right]+\frac{U_{k k}}{2} \cdot \mathbb{E}\left[(k-K)^{2}\right] .
$$

At the efficient allocation, $k-\kappa^{*}=\sum_{n \in \bar{N}^{*}} \kappa_{n}^{*}\left[\left(1-\gamma_{n}^{*}\right)\left(x_{n}-\theta_{n}\right)+\gamma_{n}^{*}\left(z_{n}-\theta_{n}\right)\right]$ implying that $\mathbb{E} k=\mathbb{E} K=\mathbb{E} \kappa^{*}$ and therefore $\mathbb{E}\left[\left(K-\kappa^{*}\right)^{2}\right]=\operatorname{Var}\left(K-\kappa^{*}\right)$ and $\mathbb{E}\left[(k-K)^{2}\right]=\operatorname{Var}(k-K)$, which gives the result.

Proof of Proposition 5. The result follows directly from the proof of Proposition 4 together with the definitions of $\kappa(\cdot), \kappa^{*}(\cdot), \alpha$ and $\alpha^{*}$.

Proof of Proposition 6. Suppose $\kappa(\cdot)=\kappa^{*}(\cdot)$ and $\alpha=\alpha^{*}$ and consider the set $\mathcal{K}$ of allocations that satisfy

$$
k(x, y)=\mathbb{E}\left[\left(1-\alpha^{\prime}\right) \kappa+\alpha^{\prime} K \mid x, y\right]
$$

for some $\alpha^{\prime}<1$, or equivalently $k(x, y)=\kappa_{0}+\sum_{n \in \bar{N}} \kappa_{n}\left[\left(1-\gamma_{n}^{\prime}\right) x_{n}+\gamma_{n}^{\prime} z_{n}\right]$, where

$$
\gamma_{n}^{\prime}=\delta_{n}+\frac{\alpha^{\prime} \delta_{n}\left(1-\delta_{n}\right)}{1-\alpha^{\prime}\left(1-\delta_{n}\right)} \quad \text { for all } n \in \bar{N}
$$

Clearly, the equilibrium (and efficient) allocation is nested with $\alpha^{\prime}=\alpha\left(=\alpha^{*}\right)$. Since for any any allocation in $\mathcal{K} \mathbb{E} K=\mathbb{E} k=\mathbb{E} \kappa$, ex-ante welfare can be written as $\mathbb{E} u=\mathbb{E} W(\kappa, \theta)-\mathcal{L}$, where

$$
\mathcal{L}=\frac{\left|W_{K K}\right|}{2} \operatorname{Var}(K-\kappa)+\frac{\left|U_{k k}+2 U_{\sigma_{k}^{2}}\right|}{2} \operatorname{Var}(k-K)=\frac{\left|U_{k k}+2 U_{\sigma_{k}^{2}}\right|}{2} \Omega,
$$

with

$$
\Omega \equiv\left(1-\alpha^{*}\right) \operatorname{Var}(K-\kappa)+\operatorname{Var}(k-K)
$$

Using

$$
\begin{aligned}
\operatorname{Var}(K-\kappa) & =\sum_{n \in \bar{N}} \kappa_{n}^{2} \gamma_{n}^{\prime 2} \sigma_{z_{n}}^{2}=\sum_{n \in \bar{N}} \kappa_{n}^{2} \gamma_{n}^{\prime 2}\left(\sigma_{n}^{2} / \delta_{n}\right), \\
\operatorname{Var}(k-K) & =\sum_{n \in \bar{N}} \kappa_{n}^{2}\left[\left(1-\gamma_{n}^{\prime}\right)^{2} \sigma_{x_{n}}^{2}\right]=\sum_{n \in \bar{N}} \kappa_{n}^{2}\left[\left(1-\gamma_{n}^{\prime}\right)^{2}\left(\sigma_{n}^{2} /\left(1-\delta_{n}\right)\right)\right]
\end{aligned}
$$

we have that

$$
\Omega=\sum_{n \in \bar{N}} \kappa_{n}^{2}\left\{\frac{\left(1-\alpha^{*}\right) \gamma_{n}^{\prime 2}}{\delta_{n}}+\frac{\left(1-\gamma_{n}^{\prime}\right)^{2}}{1-\delta_{n}}\right\} \sigma_{n}^{2} .
$$

Note that $\mathbb{E} u$ depends on $\alpha^{\prime}$ and $\left(\delta_{n}, \sigma_{n}\right)$, for $n \in \bar{N}$, only through $\Omega$. Since the efficient allocation is nested with $\alpha^{\prime}=\alpha^{*}$, it must be that $\alpha^{\prime}=\alpha^{*}$ maximizes $\mathbb{E} u$, or equivalently that $\gamma_{n}^{\prime}=\gamma_{n}^{*}$ solves $\partial \Omega / \partial \gamma_{n}^{\prime}=0$; that is,

$$
\left(1-\alpha^{*}\right) \frac{\gamma_{n}^{*}}{\delta_{n}}=\frac{1-\gamma_{n}^{*}}{1-\delta_{n}}
$$


Next note that $\Omega$ increases, and hence $\mathbb{E} u$ decreases, with any $\sigma_{n}$. Finally, consider the effect of $\delta_{n}$. By the envelope theorem,

$$
\frac{d \Omega}{d \delta_{n}}=\left.\frac{\partial \Omega}{\partial \delta_{n}}\right|_{\gamma_{n}^{\prime}=\gamma_{n}^{*}}=\kappa_{n}^{2}\left\{-\frac{\left(1-\alpha^{*}\right) \gamma_{n}^{* 2}}{\delta_{n}^{2}}+\frac{\left(1-\gamma_{n}^{*}\right)^{2}}{\left(1-\delta_{n}\right)^{2}}\right\} \sigma_{n}^{2}
$$

Using (26), we thus have that $d \mathbb{E} u / d \delta_{n}>[<] 0$ if and only if $\gamma_{n}^{*} /\left(1-\gamma_{n}^{*}\right)>[<] \delta_{n} /\left(1-\delta_{n}\right)$, which is the case if and only if $\alpha^{*}>[<] 0$. Using $\alpha=\alpha^{*}$ (by efficiency) then gives the result.

Proof of Condition (19). Since $U\left(k, K, \theta, \sigma_{k}^{2}\right)$ is quadratic in $k$ and linear in $\sigma_{k}^{2}$,

$$
U\left(k, K, \theta, \sigma_{k}^{2}\right)=U(K, K, \theta, 0)+U_{k}(K, K, \theta, 0)(k-K)+\frac{U_{k k}}{2}(k-K)^{2}+U_{\sigma_{k}^{2}} \sigma_{k}^{2} .
$$

Using the fact that $\sigma_{k}^{2}=\mathbb{E}\left[(k-K)^{2} \mid \theta, \varepsilon\right]$ and hence $\mathbb{E} \sigma_{k}^{2}=\mathbb{E}\left[(k-K)^{2}\right]$, we have that ex-ante utility is given

$$
\mathbb{E} u=\mathbb{E} W(K, \theta)+\frac{U_{k k}+2 U_{\sigma_{k}^{2}}}{2} \cdot \mathbb{E}\left[(k-K)^{2}\right] .
$$

A Taylor expansion of $W(K, \theta)$ around $K=\kappa$ then gives

$$
W(K, \theta)=W(\kappa, \theta)+W_{K}(\kappa, \theta)(K-\kappa)+\frac{W_{K K}}{2}(K-\kappa)^{2}
$$

and hence

$$
\mathbb{E} u=\mathbb{E} W(\kappa, \theta)+\mathbb{E}\left[W_{K}(\kappa, \theta) \cdot(K-\kappa)\right]+\frac{W_{K K}}{2} \cdot \mathbb{E}\left[(K-\kappa)^{2}\right]+\frac{U_{k k}+2 U_{\sigma_{k}^{2}}}{2} \cdot \mathbb{E}\left[(k-K)^{2}\right] .
$$

In equilibrium, $\mathbb{E} k=\mathbb{E} K=\mathbb{E} \kappa$ and therefore, $\mathbb{E}\left[W_{K}(\kappa, \theta) \cdot(K-\kappa)\right]=\operatorname{Cov}\left[W_{K}(\kappa, \theta),(K-\kappa)\right]$, $\mathbb{E}[(K-\kappa)]^{2}=\operatorname{Var}(K-\kappa)$ and $\mathbb{E}\left[(k-K)^{2}\right]=\operatorname{Var}(k-K)$, which gives the result.

Proof of Proposition 7. We prove the result in three steps. Step 1 computes the welfare losses due to incomplete information. Step 2 derives the comparative statics. Step 3 characterizes the bounds $\underline{\phi}, \bar{\phi}, \underline{\phi^{\prime}}, \bar{\phi}^{\prime}$.

Step 1. The property that $W$ is quadratic, along with $W_{K}\left(\kappa^{*}, \theta\right)=0$ (by definition of the first best), and $W_{K K}<0$, imply that

$$
W_{K}(\kappa, \theta)=W_{K}\left(\kappa^{*}, \theta\right)+W_{K K} \cdot\left(\kappa-\kappa^{*}\right)=\left|W_{K K}\right| \cdot\left(\kappa^{*}-\kappa\right) .
$$

It follows that

$$
\operatorname{Cov}\left(K-\kappa, W_{K}(\kappa, \theta)\right)=\left|W_{K K}\right| \cdot \operatorname{Cov}\left(K-\kappa, \kappa^{*}-\kappa\right) .
$$

Since $K-\kappa=\sum \kappa_{n} \gamma_{n}\left(z_{n}-\theta_{n}\right), z_{n}-\theta_{n}=\left[\lambda_{n}\left(\varepsilon_{n}\right)+\left(1-\lambda_{n}\right)\left(\mu_{\theta_{n}}-\theta_{n}\right)\right]$, and $\left(\varepsilon_{n}, \varepsilon_{j}, \theta_{n}, \theta_{j}\right)$ are mutually orthogonal whenever $n \neq j$, we have

$$
\begin{aligned}
\operatorname{Cov}\left(K-\kappa, \kappa^{*}-\kappa\right) & =\operatorname{Cov}\left(\sum \kappa_{n} \gamma_{n}\left(z_{n}-\theta_{n}\right), \sum\left(\kappa_{n}^{*}-\kappa_{n}\right) \theta_{n}\right)= \\
& =\sum\left(\kappa_{n}^{*}-\kappa_{n}\right) \kappa_{n} \gamma_{n} \operatorname{Cov}\left(\theta_{n}, z_{n}-\theta_{n}\right)= \\
& =\sum_{n \in \bar{N}}\left(\frac{\kappa_{n}^{*}-\kappa_{n}}{\kappa_{n}}\right) \kappa_{n}^{2} \gamma_{n}\left[-\left(1-\lambda_{n}\right) \operatorname{Var}\left(\theta_{n}\right)\right]
\end{aligned}
$$


Using $\phi_{n} \equiv\left(\kappa_{n}^{*}-\kappa_{n}\right) / \kappa_{n}, \gamma_{n}=\delta_{n} /\left(1-\alpha+\alpha \delta_{n}\right)$, and $\left(1-\lambda_{n}\right) \operatorname{Var}\left(\theta_{n}\right)=\left(\sigma_{\theta_{n}}^{-2} / \sigma_{z_{n}}^{-2}\right) \sigma_{\theta_{n}}^{2}=\sigma_{z_{n}}^{2}=$ $\sigma_{n}^{2} / \delta_{n}$, we have that

$$
\operatorname{Cov}\left(K-\kappa, \kappa^{*}-\kappa\right)=\sum_{n \in \bar{N}} \phi_{n}\left\{-\frac{1}{1-\alpha+\alpha \delta_{n}} \kappa_{n}^{2} \sigma_{n}^{2}\right\}
$$

while

$$
\operatorname{Cov}\left(K-\kappa, \kappa \mid \theta_{-n}\right)=\kappa_{n}^{2} \gamma_{n} \operatorname{Cov}\left(z_{n}-\theta_{n}, \theta_{n}\right)=-\frac{1}{1-\alpha+\alpha \delta_{n}} \kappa_{n}^{2} \sigma_{n}^{2} .
$$

Next, as in the proof of Proposition 2,

$$
\begin{aligned}
& \operatorname{Var}(K-\kappa)=\sum_{n \in \bar{N}} \frac{\delta_{n}}{\left(1-\alpha+\alpha \delta_{n}\right)^{2}} \kappa_{n}^{2} \sigma_{n}^{2} \\
& \operatorname{Var}(k-K)=\sum_{n \in \bar{N}} \frac{(1-\alpha)^{2}\left(1-\delta_{n}\right)}{\left(1-\alpha+\alpha \delta_{n}\right)^{2}} \kappa_{n}^{2} \sigma_{n}^{2} .
\end{aligned}
$$

Substituting (27)-(30) into (19), using $v=\left(1-\alpha^{*}\right)\left|U_{k k}+2 U_{\sigma_{k}^{2}}\right|$, and rearranging, we get

$$
\mathcal{L}=\frac{\left|U_{k k}+2 U_{\sigma_{k}^{2}}\right|}{2} \sum_{n \in \bar{N}} \Lambda\left(\alpha, \alpha^{*}, \phi_{n}, \delta_{n}\right) \kappa_{n}^{2} \sigma_{n}^{2}
$$

where

$$
\Lambda\left(\alpha, \alpha^{*}, \phi, \delta\right) \equiv \frac{\left(1-\alpha^{*}\right)[2 \phi(1-\alpha+\alpha \delta)+\delta]+(1-\alpha)^{2}(1-\delta)}{(1-\alpha+\alpha \delta)^{2}} .
$$

Step 2. Note that $\mathbb{E} W(\kappa, \theta)$ is independent of $\left(\delta_{n}, \sigma_{n}\right)$ and hence the comparative statics of welfare with respect to $\left(\delta_{n}, \sigma_{n}\right)$ coincide with the opposite of those of $\mathcal{L}$. Note that

$$
\begin{aligned}
\frac{\partial \mathcal{L}}{\partial \sigma_{n}^{2}} & =\frac{\left|U_{k k}+2 U_{\sigma_{k}^{2}}\right|}{2} \kappa_{n}^{2} \Lambda\left(\alpha, \alpha^{*}, \phi_{n}, \delta_{n}\right) \\
\frac{\partial \mathcal{L}}{\partial \delta_{n}} & =\frac{\left|U_{k k}+2 U_{\sigma_{k}^{2}}\right|}{2} \kappa_{n}^{2} \sigma_{n}^{2} \frac{\partial \Lambda\left(\alpha, \alpha^{*}, \phi_{n}, \delta_{n}\right)}{\partial \delta_{n}}
\end{aligned}
$$

We thus need to understand the sign of $\Lambda$ and $\partial \Lambda / \partial \delta_{n}$.

By (31),

$$
\frac{\partial \Lambda}{\partial \delta_{n}}=\frac{\alpha^{2}\left[\left(1-\delta_{n}\right)(1-\alpha)-\delta_{n}\right]-\alpha^{*}\left(1-\alpha-\alpha \delta_{n}\right)-2 \alpha \phi\left(1-\alpha^{*}\right)\left(1-\alpha+\alpha \delta_{n}\right)}{\left(1-\alpha+\alpha \delta_{n}\right)^{3}}
$$

When $\alpha=0$, this reduces to

$$
\frac{\partial \Lambda}{\partial \delta_{n}}=-\alpha^{*}
$$

and hence, for any $n \in \bar{N}, \partial \mathcal{L} / \partial \delta_{n}>[<] 0$ if and only if $\alpha^{*}<[>] 0$.

When instead $\alpha \neq 0$,

$$
\frac{\partial \Lambda}{\partial \delta_{n}}=\frac{2\left(1-\alpha^{*}\right)}{\left[1-\alpha+\alpha \delta_{n}\right]^{2}} \alpha\left[f\left(\alpha, \alpha^{*}, \delta_{n}\right)-\phi_{n}\right]
$$


where

$$
f\left(\alpha, \alpha^{*}, \delta\right) \equiv \frac{\alpha^{2}[(1-\delta)(1-\alpha)-\delta]-\alpha^{*}(1-\alpha-\alpha \delta)}{2 \alpha(1-\alpha+\alpha \delta)\left(1-\alpha^{*}\right)} .
$$

Since $\alpha^{*}<1, \operatorname{sign}\left[\partial \mathcal{L} / \partial \delta_{n}\right]=\operatorname{sign}[\alpha] \cdot \operatorname{sign}\left[f\left(\alpha, \alpha^{*}, \delta_{n}\right)-\phi_{n}\right]$. Let

$$
\underline{\phi}\left(\alpha, \alpha^{*}\right) \equiv \min _{\delta \in[0,1]} f\left(\alpha, \alpha^{*}, \delta\right) \quad \text { and } \quad \bar{\phi}\left(\alpha, \alpha^{*}\right) \equiv \max _{\delta \in[0,1]} f\left(\alpha, \alpha^{*}, \delta\right) .
$$

If $\phi_{n} \in(\underline{\phi}, \bar{\phi})$, then $\partial \mathcal{L} / \partial \delta_{n}$ alternates sign as $\delta_{n}$ varies between 0 and 1 , no matter whether $\alpha>0$ or $\alpha<0$. Hence, $\phi_{n}<\underline{\phi}$ is necessary and sufficient for $\partial \mathcal{L} / \partial \delta_{n}>0 \forall \delta_{n}$ when $\alpha>0$ and for $\partial \mathcal{L} / \partial \delta_{n}<0 \forall \delta_{n}$ when $\alpha<0$, whereas $\phi_{n}>\bar{\phi}$ is necessary and sufficient for $\partial \mathcal{L} / \partial \delta_{n}<0 \forall \delta_{n}$ when $\alpha>0$ and for $\partial \mathcal{L} / \partial \delta_{n}>0 \forall \delta_{n}$ when $\alpha<0$.

Finally, note that $\partial \mathcal{L} / \partial \sigma_{n}^{2}>[<] 0$ if and only if $\phi_{n}>[<] g\left(\alpha, \alpha^{*}, \delta_{n}\right)$, where

$$
g\left(\alpha, \alpha^{*}, \delta\right)=-\frac{(1-\alpha)^{2}(1-\delta)+\delta\left(1-\alpha^{*}\right)}{2\left(1-\alpha^{*}\right)(1-\alpha+\alpha \delta)}<0 .
$$

Letting

$$
\underline{\phi^{\prime}}\left(\alpha, \alpha^{*}\right) \equiv \min _{\delta \in[0,1]} g\left(\alpha, \alpha^{*}, \delta\right) \quad \text { and } \quad \bar{\phi}^{\prime}\left(\alpha, \alpha^{*}\right) \equiv \max _{\delta \in[0,1]} g\left(\alpha, \alpha^{*}, \delta\right),
$$

we get that $\partial \mathcal{L} / \partial \sigma_{n}^{2}>0[<0]$ for all $\delta_{n} \in[0,1]$ if $\phi_{n}>\bar{\phi}^{\prime}\left[<\underline{\phi}^{\prime}\right]$, whereas $\partial \mathcal{L} / \partial \sigma_{n}^{2}$ alternates sign as $\delta_{n}$ varies if $\phi_{n} \in\left(\underline{\phi}^{\prime}, \bar{\phi}^{\prime}\right)$.

Step 3. Note that both $f$ and $g$ are monotonic in $\delta$, with

$$
\frac{\partial f}{\partial \delta}=-2 \frac{\partial g}{\partial \delta}=\frac{(1-\alpha)}{\left(1-\alpha^{*}\right)(1-\alpha+\alpha \delta)^{2}}\left(\alpha^{*}-\alpha\right)
$$

When $\alpha^{*}=\alpha$, both $f$ and $g$ are independent of $\delta$, and

$$
\underline{\phi^{\prime}}\left(\alpha, \alpha^{*}\right)=\underline{\phi}\left(\alpha, \alpha^{*}\right)=\bar{\phi}\left(\alpha, \alpha^{*}\right)=\bar{\phi}^{\prime}\left(\alpha, \alpha^{*}\right)=-\frac{1}{2}<0 .
$$

When instead $\alpha^{*}>\alpha, f$ is strictly increasing (and $g$ strictly decreasing) in $\delta$, so that

$$
\begin{aligned}
\underline{\phi}\left(\alpha, \alpha^{*}\right) & =f\left(\alpha, \alpha^{*}, 0\right)<\bar{\phi}\left(\alpha, \alpha^{*}\right)=f\left(\alpha, \alpha^{*}, 1\right), \\
\underline{\phi}^{\prime}\left(\alpha, \alpha^{*}\right) & =g\left(\alpha, \alpha^{*}, 1\right)<\bar{\phi}^{\prime}\left(\alpha, \alpha^{*}\right)=g\left(\alpha, \alpha^{*}, 0\right),
\end{aligned}
$$

and when $\alpha^{*}<\alpha, f$ is strictly decreasing in $\delta$ and

$$
\begin{aligned}
\underline{\phi}\left(\alpha, \alpha^{*}\right) & =f\left(\alpha, \alpha^{*}, 1\right)<\bar{\phi}\left(\alpha, \alpha^{*}\right)=f\left(\alpha, \alpha^{*}, 0\right) \\
\underline{\phi}^{\prime}\left(\alpha, \alpha^{*}\right) & =g\left(\alpha, \alpha^{*}, 0\right)<\bar{\phi}^{\prime}\left(\alpha, \alpha^{*}\right)=g\left(\alpha, \alpha^{*}, 1\right) .
\end{aligned}
$$

Consider first the case $\alpha \in(0,1)$. If $\alpha^{*}>\alpha$, then $\alpha^{2}+(1-2 \alpha) \alpha^{*}>0$ (using the fact that $\left.\alpha^{*}<1\right)$ and therefore

$$
\underline{\phi}\left(\alpha, \alpha^{*}\right)<\bar{\phi}\left(\alpha, \alpha^{*}\right)=f\left(\alpha, \alpha^{*}, 1\right)=-\frac{\alpha^{2}+(1-2 \alpha) \alpha^{*}}{2 \alpha\left(1-\alpha^{*}\right)}<0 .
$$


If instead $\alpha^{*}<\alpha$, then

$$
\underline{\phi}\left(\alpha, \alpha^{*}\right)=f\left(\alpha, \alpha^{*}, 1\right)=-\frac{\alpha^{2}+(1-2 \alpha) \alpha^{*}}{2 \alpha\left(1-\alpha^{*}\right)}<\bar{\phi}\left(\alpha, \alpha^{*}\right)=f\left(\alpha, \alpha^{*}, 0\right)=-\frac{\alpha^{*}-\alpha^{2}}{2 \alpha\left(1-\alpha^{*}\right)}
$$

and therefore $\underline{\phi}<0$ if and only if $\alpha>1 / 2$ or $\alpha^{*}>-\alpha^{2} /(1-2 \alpha)$, while $\bar{\phi}<0$ if and only if $\alpha^{*}>\alpha^{2}$. Since $-\alpha^{2} /(1-2 \alpha)<0$ whenever $\alpha<1 / 2$, we conclude that, for $\alpha \in(0,1), \underline{\phi}<0$ if and only if $\alpha>1 / 2$ or $\alpha^{*}>-\alpha^{2} /(1-2 \alpha)$, and $\bar{\phi}<0$ if and only if $\alpha^{*}>\alpha^{2}$.

Next, consider the case $\alpha \in(-1,0)$. If $\alpha^{*}>\alpha$, then

$$
\underline{\phi}\left(\alpha, \alpha^{*}\right)=f\left(\alpha, \alpha^{*}, 0\right)=\frac{\alpha^{*}-\alpha^{2}}{(-2 \alpha)\left(1-\alpha^{*}\right)}<\bar{\phi}\left(\alpha, \alpha^{*}\right)=f\left(\alpha, \alpha^{*}, 1\right)=\frac{\alpha^{2}+(1-2 \alpha) \alpha^{*}}{(-2 \alpha)\left(1-\alpha^{*}\right)}
$$

and hence $\underline{\phi}<0$ if and only if $\alpha^{*}<\alpha^{2}$, while $\bar{\phi}<0$ if and only if $\alpha^{*}<-\alpha^{2} /(1-2 \alpha)$. If instead $\alpha^{*}<\alpha$, then $\alpha^{*}<0<\alpha^{2}$ and hence

$$
\underline{\phi}\left(\alpha, \alpha^{*}\right)<\bar{\phi}\left(\alpha, \alpha^{*}\right)=f\left(\alpha, \alpha^{*}, 0\right)=\frac{\alpha^{*}-\alpha^{2}}{(-2 \alpha)\left(1-\alpha^{*}\right)}<0 .
$$

We conclude that, for $\alpha \in(0,1), \underline{\phi}<0$ if and only if $\alpha^{*}<\alpha^{2}$, and $\bar{\phi}<0$ if and only if $\alpha^{*}<$ $-\alpha^{2} /(1-2 \alpha)$.

Finally, note that

$$
g\left(\alpha, \alpha^{*}, 0\right)=-\frac{(1-\alpha)}{2\left(1-\alpha^{*}\right)}<0 \quad \text { and } \quad g\left(\alpha, \alpha^{*}, 1\right)=-\frac{1}{2}<0
$$

Together with the monotonicity of $g$, this implies that $\underline{\phi}^{\prime} \leq \bar{\phi}^{\prime}<0$ for all $\left(\alpha, \alpha^{*}\right), \bar{\phi}^{\prime}<-1 / 2$ whenever $\alpha<\alpha^{*}$, and $\underline{\phi}^{\prime}>-1 / 2$ whenever $\alpha>\alpha^{*}$.

\section{References}

[1] Acemoglu, Daron (1993), "Learning About Others' Actions and the Investment Accelerator," Economic Journal 103, 318-328.

[2] Angeletos, George-Marios, and Alessandro Pavan (2004), "Transparency of Information and Coordination in Economies with Investment Complementarities," American Economic Review $94(\mathrm{P} \& \mathrm{P})$, 91-98.

[3] Angeletos, George-Marios, and Ivàn Werning (2004), "Crises and Prices: Information Aggregation, Multiplicity and Volatility," MIT mimeo.

[4] Atkeson, Andrew, and Patrick Kehoe (2001), "The Advantage of Transparent Instruments of Monetary Policy," NBER working paper. 
[5] Benhabib, Jess, and Roger Farmer (1994), "Indeterminacy and Increasing Returns," Journal of Economic Theory 63, 19-41.

[6] Bryant, John (1983), "A Simple Rational Expectations Keynes-Type Model," Quarterly Journal of Economics 98, 3, 525-28.

[7] Canzoneri, Matthew (1985), "Monetary Policy Games and the Role of Private Information," American Economic Review 75, 1056-70.

[8] Cooper, Russell, and Andrew John (1988), "Coordinating Coordination Failures in Keynesian Models," Quarterly Journal of Economics 103, 441-463.

[9] Cukierman, Alex, and Allan Meltzer (1986), "A Theory of Ambiguity, Credibility, and Inflation under Discretion and Asymmetric Information," Econometrica 54, 1099-128.

[10] Gereatts, Petra (2002), "Central Bank Transparency," Economic Journal 112, F532-F565.

[11] Grossman, Sanford (1981) "An Introduction to the Theory of Rational Expectations under Asymmetric Information," Review of Economic Studies 48, 541-559.

[12] Heinemann, Frank, and Camille Cornand (2004), "Optimal Degree of Public Information Dissemination," CESifo Working Paper 1353.

[13] Hellwig, Christian (2005), "Heterogeneous Information and the Benefits of Transparency," UCLA mimeo.

[14] Hirshleifer, Jack (1971), "The Private and Social Value of Information and the Reward to Inventive Activity," American Economic Review 61, 561-574.

[15] Holmstrom, Bengt, and Roger Myerson (1983), "Efficient and Durable Decision Rules with Incomplete Information," Econometrica 51, 1799-1819.

[16] Laffont, Jean-Jacques (1985), "On the Welfare Analysis of Rational Expectations Equilibria with Asymmetric Information, " Econometrica 53, 1-30.

[17] Lorenzoni, Guido (2005), "Imperfect Information, Consumers' Expectations and Business Cycles," MIT mimeo.

[18] Matsuyama, Kiminori (1992), "A Simple Model of Sectoral Adjustment," The Review of Economic Studies 59, 375-388.

[19] Messner, Simon, and Xavier Vives (2001), "Informational and Economic Efficiency in REE with Asymmetric Information," CEPR working paper 2678. 
[20] Morris, Stephen, and Hyun Song Shin (2002), "The Social Value of Public Information", American Economic Review 92, 1521-1534.

[21] Morris, Stephen, and Hyun Song Shin (2005), "Central Bank Transparency and the Signal Value of Prices," forthcoming, Brookings Papers on Economic Activity.

[22] Radner, Roy (1962), "Team Decision Problems," Annals of Mathematical Statistics 33, 857881.

[23] Raith, Michael (1996), "A General Model of Information Sharing in Oligopoly," Journal of Economic Theory 71, 260-288.

[24] Roca, Mauro (2005), "Transparency and Monetary Policy with Imperfect Common Knowledge," Columbia University mimeo.

[25] Stokey, Nancy (2002), "Rules versus Discretion After Twenty-Five Years," NBER Macroeconomics Annual 17, 9-45.

[26] Svensson, Lars (2005), "The Social Value of Public Information: Morris and Shin (2002) is Actually Pro Transparency, Not Con," forthcoming in American Economic Review.

[27] Vives, Xavier (1988), "Aggregation of Information in Large Cournot Markets, " Econometrica $56,851-876$.

[28] Woodford, Michael (2003), Interest and Prices: Foundations of a Theory of Monetary Policy, Princeton University Press.

[29] Woodford, Michael (2005), "Central Bank Communication and Policy Effectiveness," paper given at the 2005 Symposium of the Federal Reserve Bank of Kansas City at Jacksons Hole, The Greenspan Era: Lessons for the Future. 